\title{
Enhanced Control of Phosphinoylcarbodithioate-Mediated RAFT Polymerization: Key Role of Substituents at the Phosphorus Center
}

\author{
Ihor Kulai, ${ }^{\dagger}$ Zoia Voitenko, ${ }^{\ddagger}$ Stephane Mazieres, ${ }^{\dagger}$ Mathias Destarac ${ }^{\dagger}$ \\ tIMRCP, UMR 5623, Université de Toulouse, 118, route de Narbonne F-31062 Toulouse, Cedex 9, France \\ ‡Taras Shevchenko National University of Kyiv, Department of Chemistry, 64/13, Volodymyrska Street, Kyiv, Ukraine \\ 01601
}

\section{LIST OF ABBREVIATIONS}

$\begin{array}{ll}\text { AIBN } & \text { Azobisisobutyronitrile; } \\ \text { BA/PBA } & \text { Butyl acrylate/Poly(butyl acrylate) } \\ \text { CEF } & \text { Chain-end fidelity; } \\ \text { CTA } & \text { Chain transfer agent; } \\ \text { ESI HRMS } & \text { Electrospray ionization high-resolution mass spectrometry; } \\ \text { IR } & \text { Infrared; } \\ \text { NMR } & \text { Nuclear magnetic resonance; } \\ \text { MALDI TOF MS } & \text { Matrix-assisted laser desorption ionization time-of-flight mass spectrometry } \\ \text { MMD } & \text { Molar mass distribution; } \\ \text { RAFT } & \text { Reversible addition-fragmentation chain transfer; } \\ \text { SEC } & \text { Size-exclusion chromatography; } \\ \text { St/PSt } & \text { Styrene/Polystyrene } \\ \text { TPPO } & \text { Triphenylphosphine oxide; } \\ \text { TPPS } & \text { Triphenylphosphine sulphide; }\end{array}$

\section{TABLE OF CONTENTS}

Table S1. Key spectral characteristics of P-RAFT 1-6.

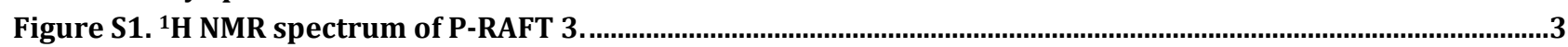

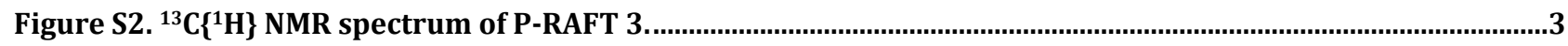

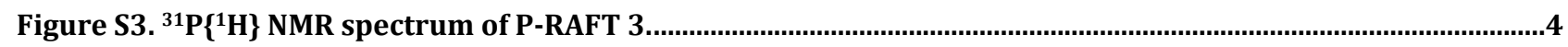

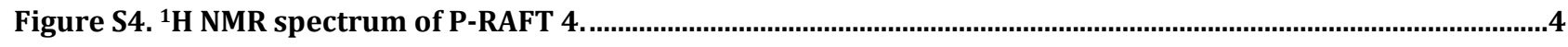

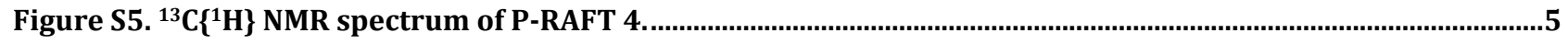

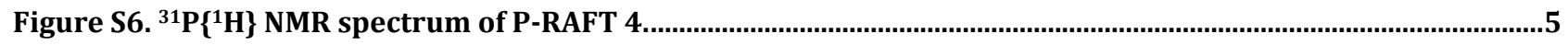

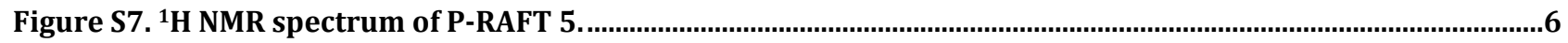

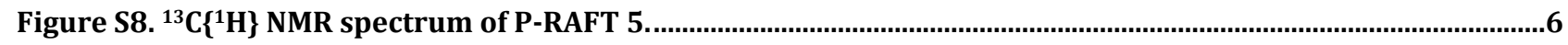

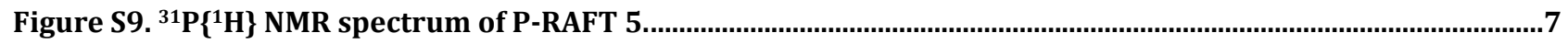

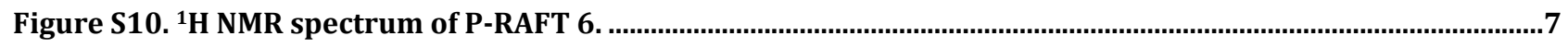

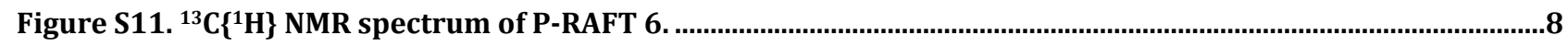

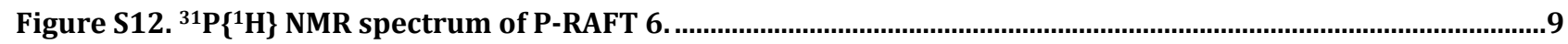

Table S2. Macromolecular characteristics of PBA samples obtained during P-RAFT mediated polymerization ..9 Figure S13. Semi-logarithmic kinetic plot for the initial period of P-RAFT 1-6 mediated BA polymerization.....10 Figure S14. Overlay of the SEC chromatograms of PBA samples obtained in P-RAFT 1 mediated polymerization.10 Figure S15. Overlay of the SEC chromatograms of PBA samples obtained in P-RAFT 2 mediated polymerization.10 Figure S16. Overlay of the SEC chromatograms of PBA samples obtained in P-RAFT 4 mediated polymerization.10 Figure S17. Overlay of the SEC chromatograms of PBA samples obtained in P-RAFT 5 mediated polymerization.10 Figure S18. Overlay of the SEC chromatograms of PBA samples obtained in P-RAFT 6 mediated polymerization.10 Figure S19. ${ }^{31} \mathrm{P}\left\{{ }^{1} \mathrm{H}\right\}$ NMR spectra of reaction mixtures obtained in P-RAFT 1 mediated polymerization of BA...11 Figure S20. ${ }^{31} \mathrm{P}\left\{{ }^{1} \mathrm{H}\right\}$ NMR spectra of reaction mixtures obtained in P-RAFT 2 mediated polymerization of BA. ..11 Figure S21. ${ }^{31} \mathrm{P}\left\{{ }^{1} \mathrm{H}\right\}$ NMR spectra of reaction mixtures obtained in P-RAFT 3 mediated polymerization of BA...12 Figure S22. ${ }^{31} \mathrm{P}\left\{{ }^{1} \mathrm{H}\right\}$ NMR spectra of reaction mixtures obtained in P-RAFT 4 mediated polymerization of BA. ..12 Figure S23. ${ }^{31} \mathrm{P}\left\{{ }^{1} \mathrm{H}\right\}$ NMR spectra of reaction mixtures obtained in P-RAFT 5 mediated polymerization of BA. ..13 Figure S24. ${ }^{31} \mathrm{P}\left\{{ }^{1} \mathrm{H}\right\}$ NMR spectra of reaction mixtures obtained in P-RAFT 6 mediated polymerization of BA. ..13 
Figure S25. MALDI-TOF MS spectra of PBA samples prepared starting from P-RAFT 1-4. .14

Table S3. Macromolecular characteristics of PSt samples obtained in P-RAFT mediated polymerization ..........15 Figure S26. Overlay of the SEC chromatograms of PSt samples obtained in P-RAFT 1 mediated polymerization.16 Figure S27. Overlay of the SEC chromatograms of PSt samples obtained in P-RAFT 2 mediated polymerization.16 Figure S28. Overlay of the SEC chromatograms of PSt samples obtained in P-RAFT 4 mediated polymerization.16 Figure S29. ${ }^{31} \mathrm{P}\left\{{ }^{1} \mathrm{H}\right\}$ NMR spectra of reaction mixtures obtained in P-RAFT 1 mediated polymerization of St.....17 Figure S30. ${ }^{31} \mathrm{P}\left\{{ }^{1} \mathrm{H}\right\}$ NMR spectra of reaction mixtures obtained in P-RAFT 2 mediated polymerization of St.....17 Figure S31. ${ }^{31} \mathrm{P}\left\{{ }^{1} \mathrm{H}\right\}$ NMR spectra of reaction mixtures obtained in P-RAFT 3 mediated polymerization of St.....18 Figure S32. ${ }^{31} \mathrm{P}\left\{{ }^{1} \mathrm{H}\right\}$ NMR spectra of reaction mixtures obtained in P-RAFT 4 mediated polymerization of St.....18 Figure S33. ${ }^{31} \mathrm{P}\left\{{ }^{1} \mathrm{H}\right\}$ NMR spectra of reaction mixtures obtained in PBA-3 mediated polymerization of St..........19 Table S4. ${ }^{31} \mathrm{P}\left\{{ }^{1} \mathrm{H}\right\}$ NMR chemical shifts used to determine the RAFT $\omega$ chain-end fidelity.

Table S1. Key spectral characteristics of P-RAFT 1-6.

\begin{tabular}{|c|c|c|c|c|c|c|}
\hline P-RAFT & $\mathbf{1}^{1}$ & $2^{1}$ & 3 & 4 & 5 & 6 \\
\hline \multicolumn{7}{|c|}{${ }^{1} \mathrm{H}$ NMR } \\
\hline$\delta_{\mathrm{CH}}, \mathrm{ppm}$ & 5.23 & 5.04 & 5.14 & 5.14 & 4.68 & 4.61 \\
\hline${ }^{3} J_{\mathrm{H}, \mathrm{H}}, \mathrm{Hz}$ & 7.2 & 7.1 & 7.1 & 7.1 & 7.3 & 7.3 \\
\hline${ }^{4} J_{\mathrm{P}, \mathrm{H}}, \mathrm{Hz}$ & 2.1 & 1.8 & 1.7 & 1.7 & 1.4 & 1.2 \\
\hline$\delta_{\mathrm{CH} 3}, \mathrm{ppm}$ & 1.74 & 1.67 & 1.67 & 1.71 & 1.58 & 1.56 \\
\hline${ }^{3} J_{\mathrm{H}, \mathrm{H}}, \mathrm{Hz}$ & 7.2 & 7.2 & 7.1 & 7.1 & 7.4 & 7.4 \\
\hline${ }^{5} J_{\mathrm{P}, \mathrm{H}}, \mathrm{Hz}$ & 0.9 & 0.7 & -- & 0.6 & 0.8 & 1.0 \\
\hline \multicolumn{7}{|c|}{${ }^{13} \mathrm{C}\left\{{ }^{1} \mathrm{H}\right\}$ NMR } \\
\hline$\delta_{\mathrm{CS} 2}, \mathrm{ppm}$ & 235.0 & 242.8 & 237.5 & 235.0 & 235.3 & 235.5 \\
\hline${ }^{1} J_{\mathrm{P}, \mathrm{C}}, \mathrm{Hz}$ & 76.1 & 126.2 & 52.8 & 130.4 & 74.8 & 130.3 \\
\hline \multicolumn{7}{|c|}{${ }^{31} \mathrm{P}\left\{{ }^{1} \mathrm{H}\right\} \mathrm{NMR}$} \\
\hline$\delta, \mathrm{ppm}$ & 24.9 & 14.9 & 51.4 & 14.1 & 22.9 & 14.1 \\
\hline \multicolumn{7}{|c|}{ IR } \\
\hline$\nu_{\mathrm{P}=0}, \mathrm{~cm}^{-1}$ & 1190.0 & 1218.0 & 1164.3 & 1217.7 & 1198.9 & 1217.9 \\
\hline$v_{\mathrm{C}=\mathrm{S}}, \mathrm{cm}^{-1}$ & 1097.1 & 1092.0 & 1081.3 & 1083.5 & 1098.9 & 1069.3 \\
\hline$v_{\mathrm{C}=0}, \mathrm{~cm}^{-1}$ & & & & & 1737.6 & 1738.7 \\
\hline \multicolumn{7}{|c|}{ HRMS } \\
\hline$m / z_{\text {measured }}$ & 383.0685 & 429.2170 & 395.1626 & 397.1538 & 365.0441 & 379.1272 \\
\hline$m / z_{\text {calculated }}$ & 383.0685 & 429.2163 & 395.1632 & 397.1537 & 365.0441 & 379.1279 \\
\hline
\end{tabular}

${ }^{1}$ Mazières, S.; Kulai, I.; Geagea, R.; Ladeira, S.; Destarac, M. Phosphinoyl and Thiophosphinoylcarbodithioates: Synthesis, Molecular Structure, and Application as New Efficient Mediators for RAFT Polymerization. Chem. - A Eur. J. 2015, 21 (4), 1726-1734 DOI: 10.1002/chem.201404631. 

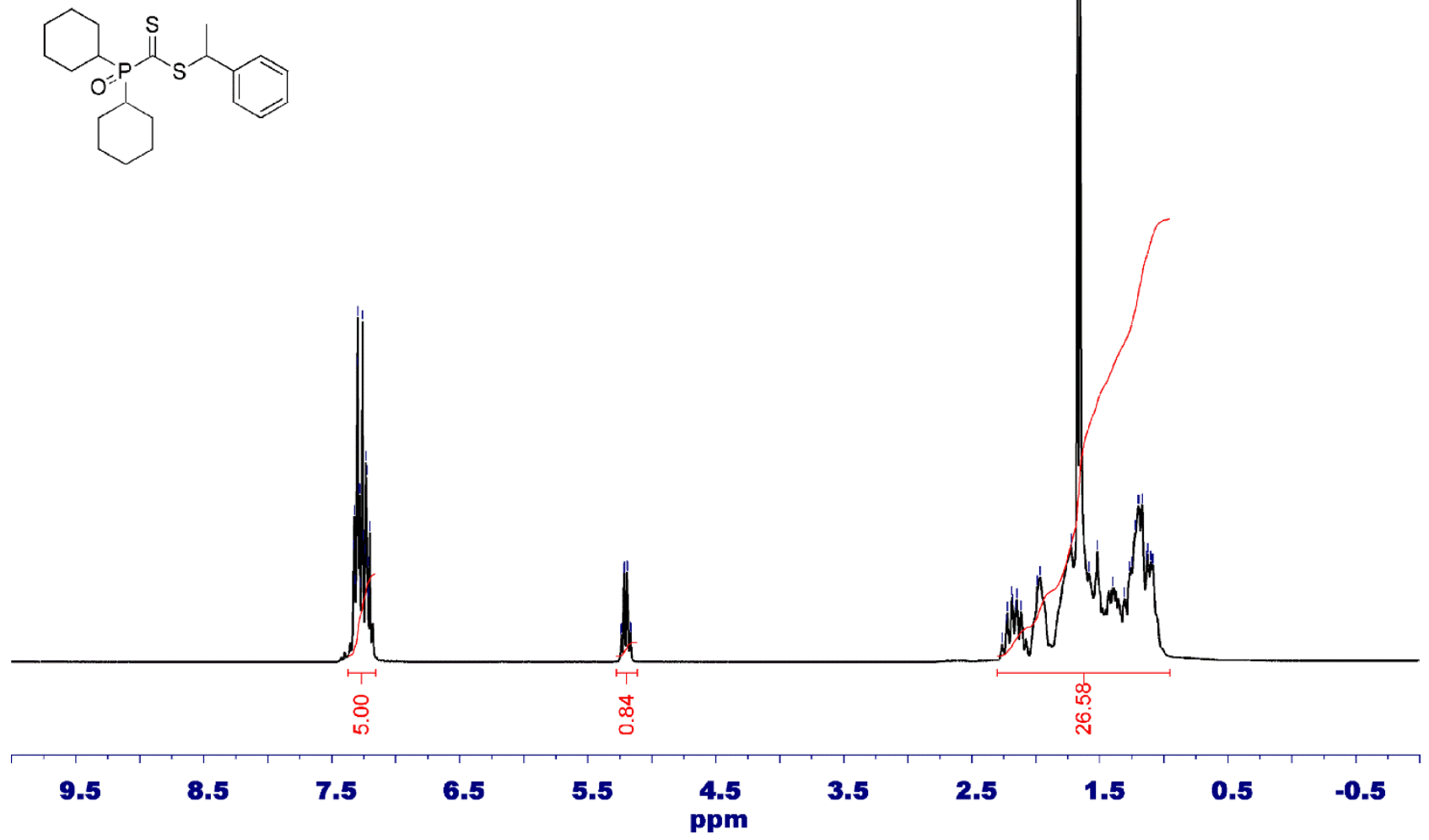

Figure S1. ${ }^{1} \mathrm{H}$ NMR spectrum of $S$-(1-phenylethyl) (dicyclohexylphosphinoyl)carbodithioate P-RAFT 3.

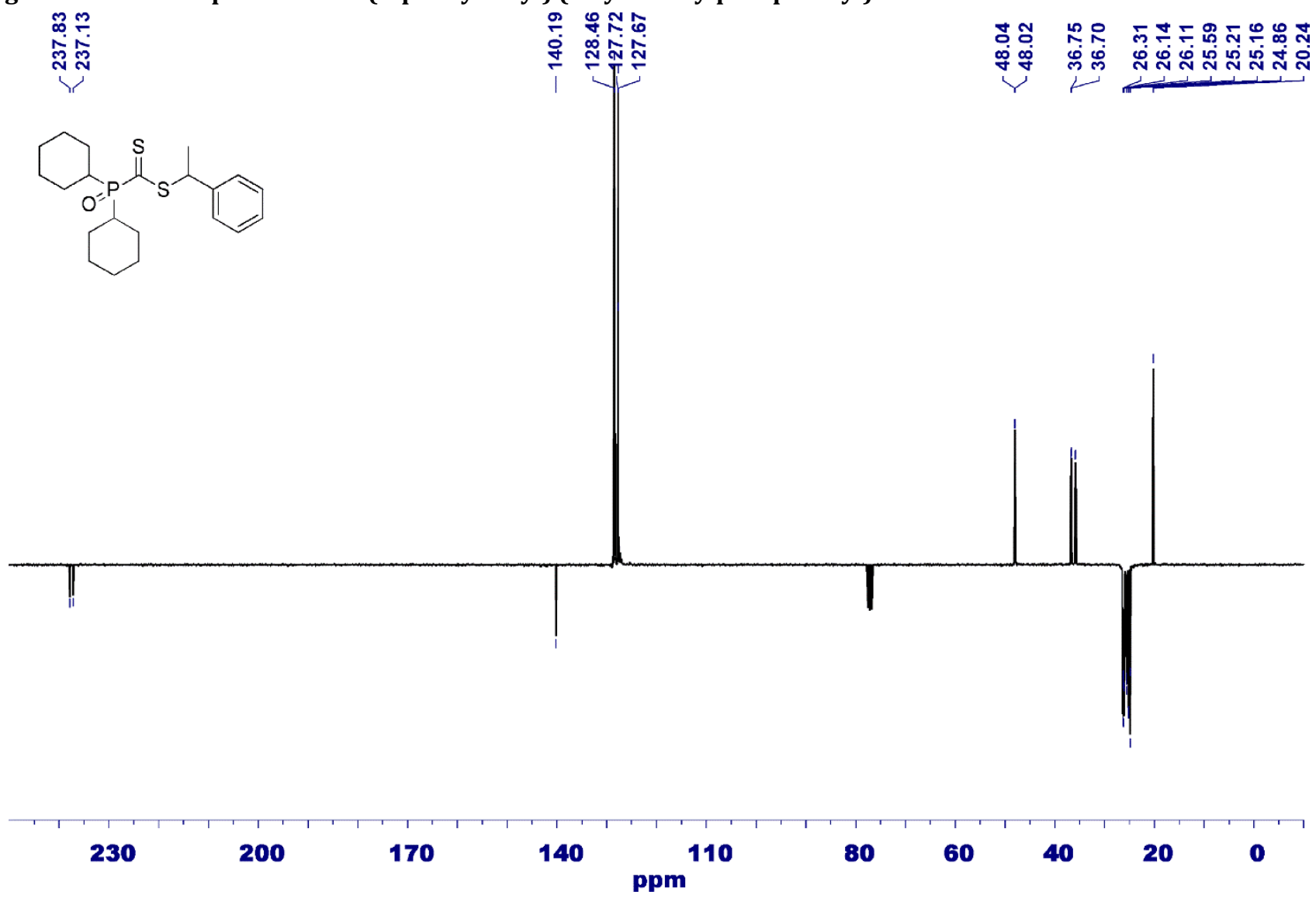

Figure S2. ${ }^{13} \mathrm{C}\left\{{ }^{1} \mathrm{H}\right\}$ NMR spectrum of $S$-(1-phenylethyl) (dicyclohexylphosphinoyl)carbodithioate P-RAFT 3. 


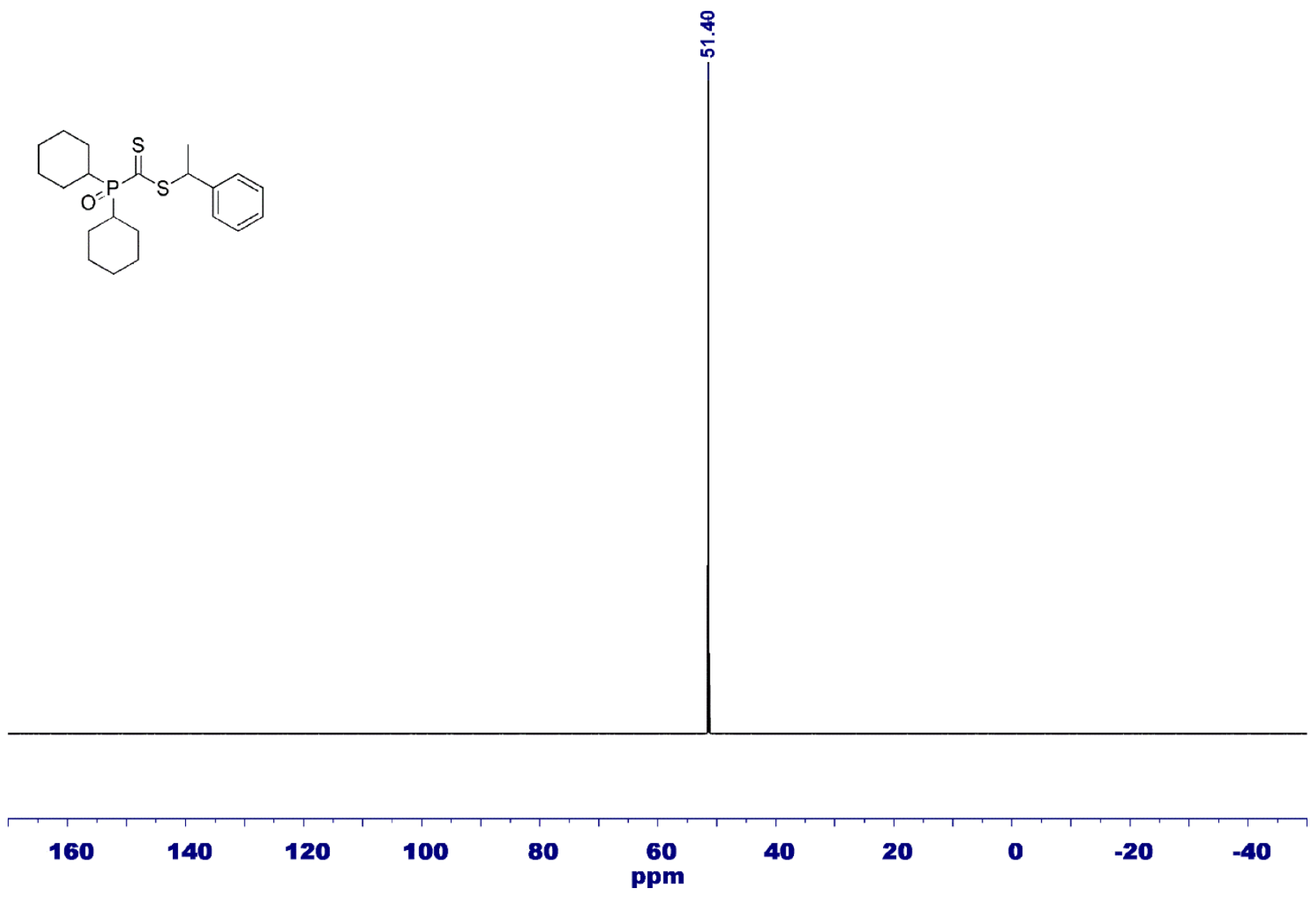

Figure S3. ${ }^{31} \mathrm{P}\left\{{ }^{1} \mathrm{H}\right\}$ NMR spectrum of $S$-(1-phenylethyl) (dicyclohexylphosphinoyl)carbodithioate P-RAFT 3.

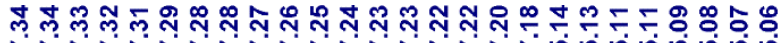

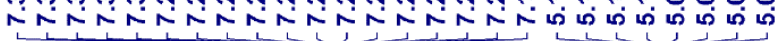

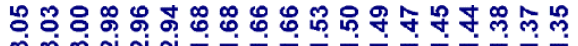
LI m地<smiles>CC(SC(=S)P(=O)(N(C)C)N1CCCCC1)c1ccccc1</smiles>
Q
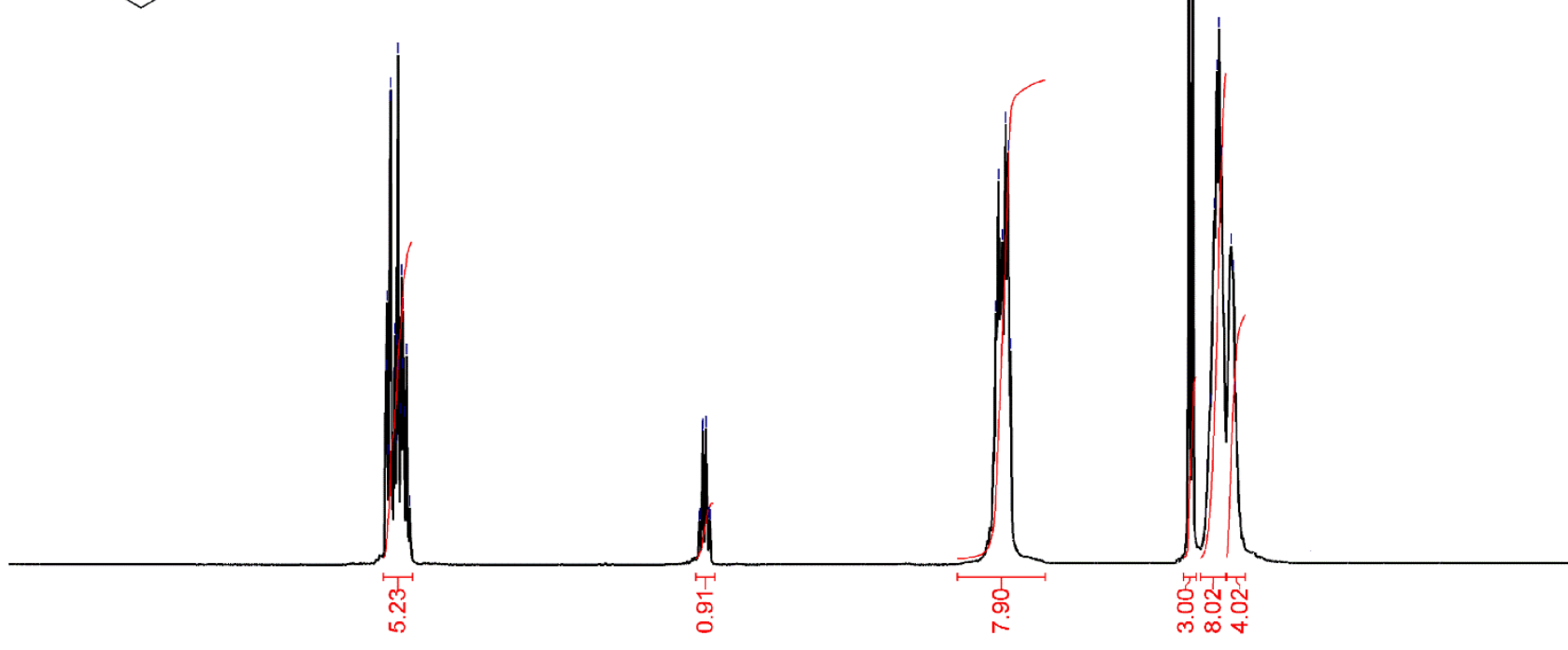

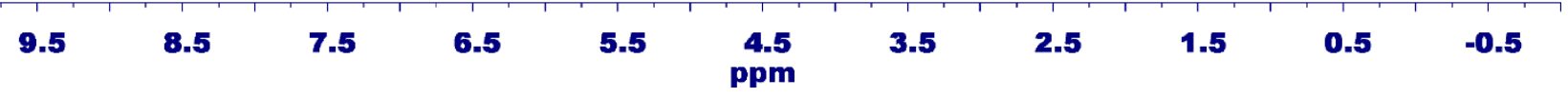

Figure S4. ${ }^{1} \mathrm{H}$ NMR spectrum of $S$-(1-phenylethyl) (di(piperidin-1-yl)phosphinoyl)carbodithioate P-RAFT 4. 


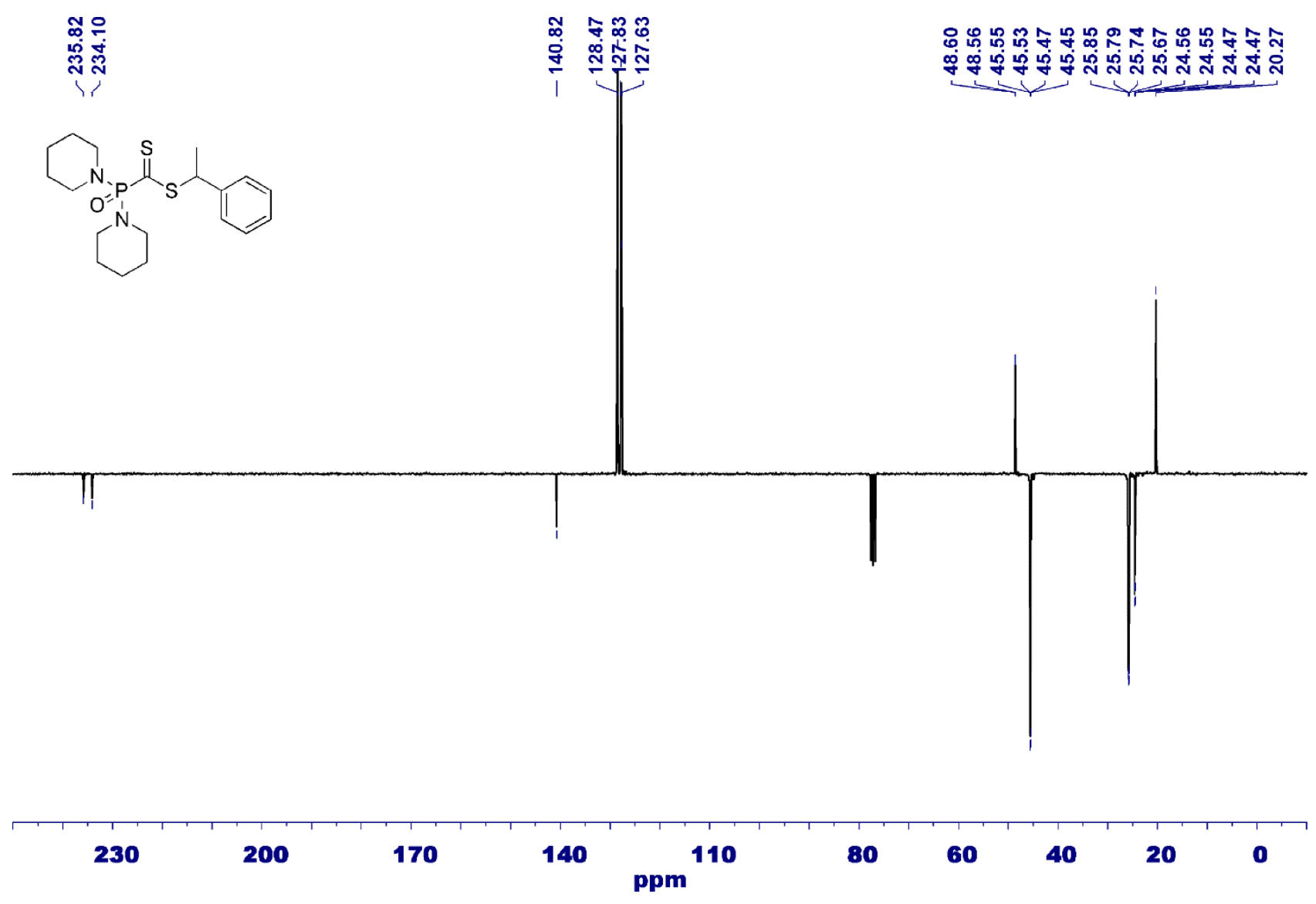

Figure S5. ${ }^{13} \mathrm{C}\left\{{ }^{1} \mathrm{H}\right\}$ NMR spectrum of $S$-(1-phenylethyl) (di(piperidin-1-yl)phosphinoyl)carbodithioate P-RAFT 4.

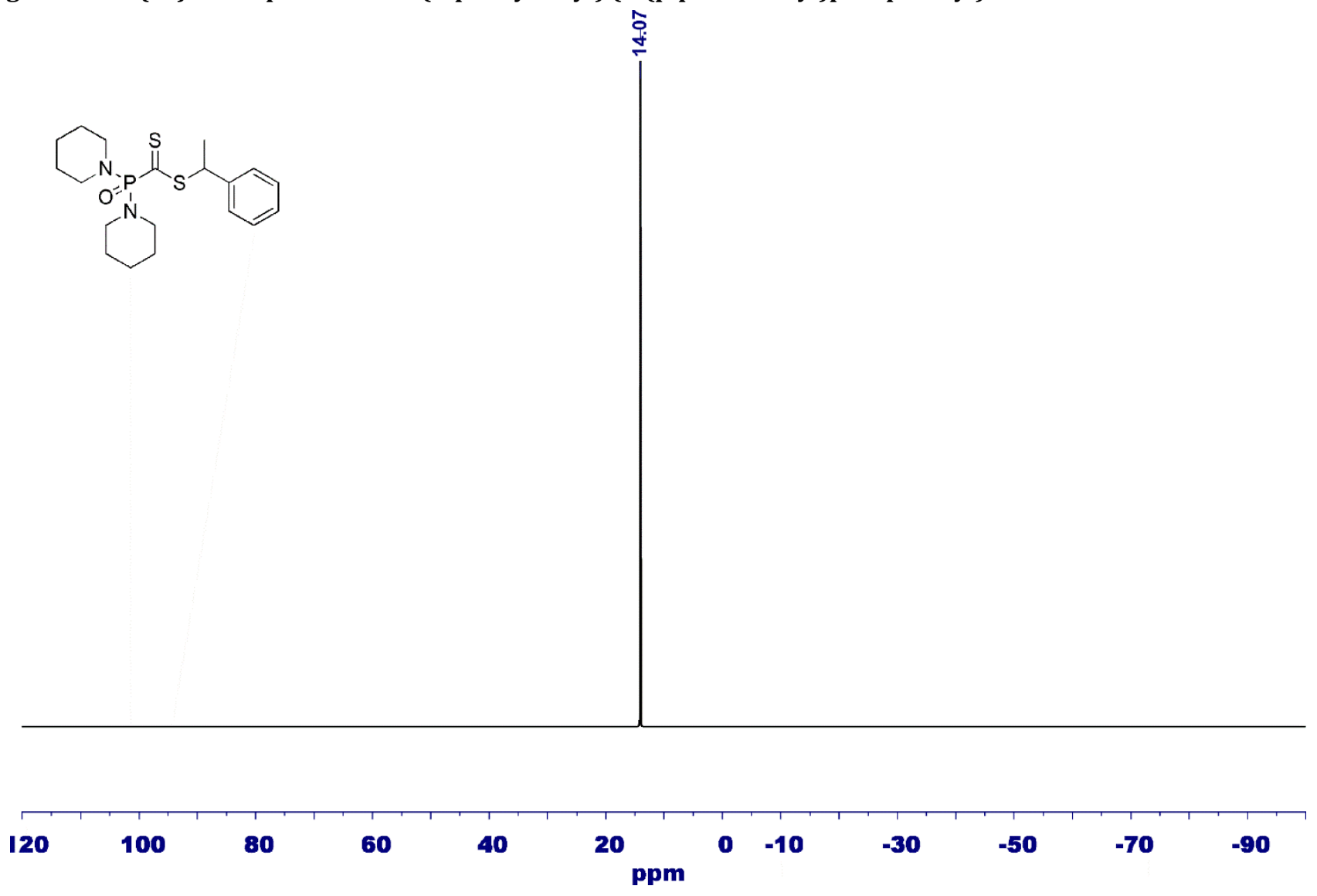

Figure S6. ${ }^{31} \mathrm{P}\left\{{ }^{1} \mathrm{H}\right\}$ NMR spectrum of $S$-(1-phenylethyl) (di(piperidin-1-yl)phosphinoyl)carbodithioate P-RAFT 4. 
<smiles>COC(=O)C(C)SC(=S)P(c1ccccc1)c1ccccc1</smiles>

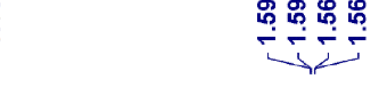

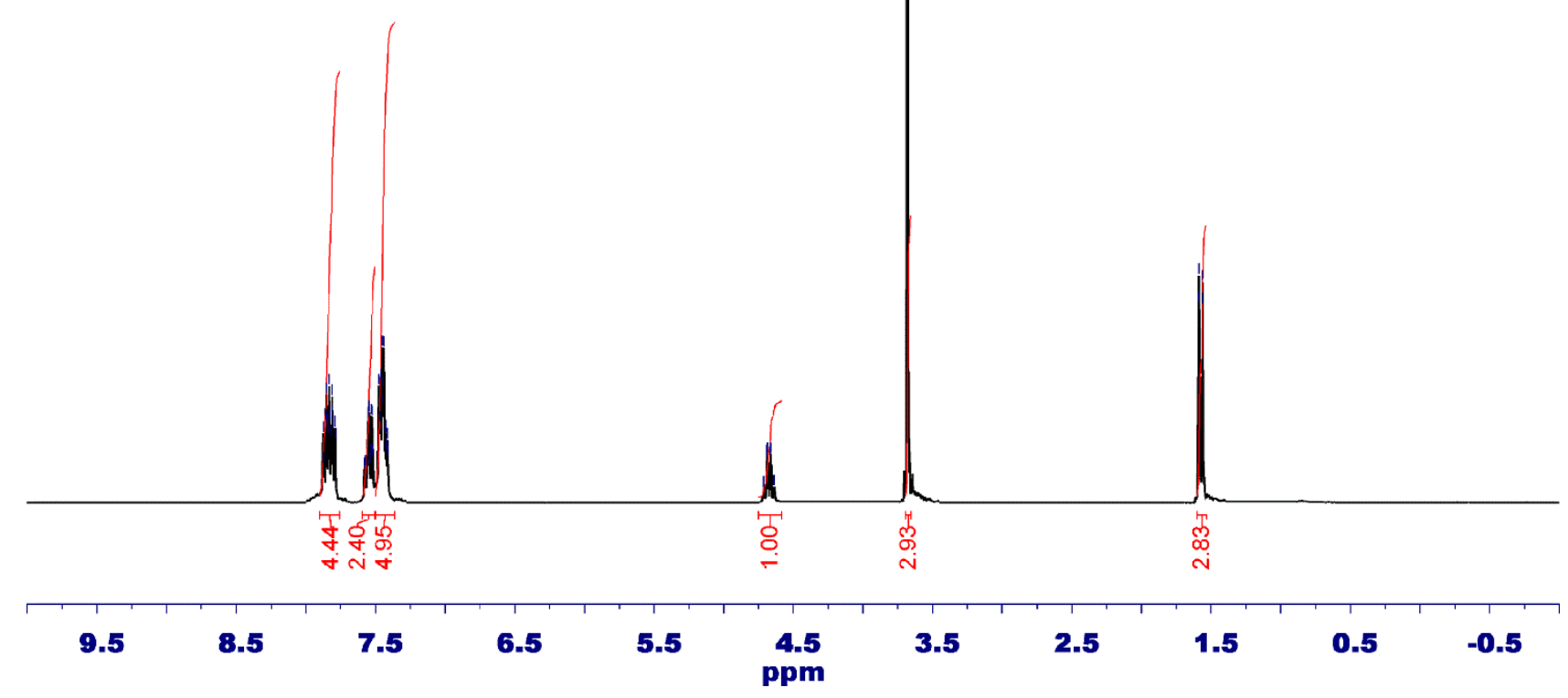

Figure S7. ${ }^{1} \mathrm{H}$ NMR spectrum of $S$-((1-methoxycarbonyl)ethyl) (diphenylphosphinoyl)carbodithioate P-RAFT 5.

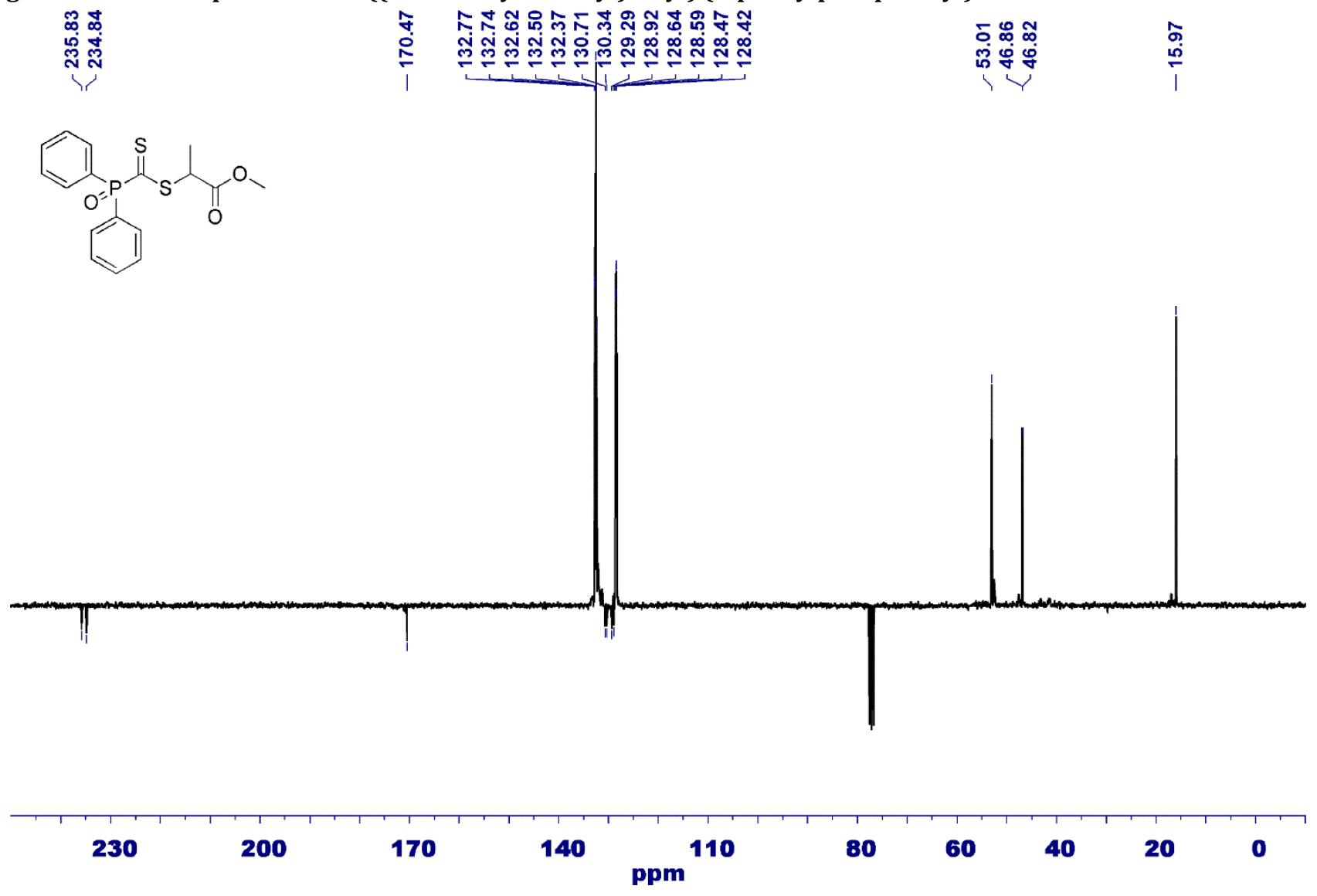

Figure S8. ${ }^{13} \mathrm{C}\left\{{ }^{1} \mathrm{H}\right\}$ NMR spectrum of $S$-((1-methoxycarbonyl)ethyl) (diphenylphosphinoyl)carbodithioate P-RAFT 5. 


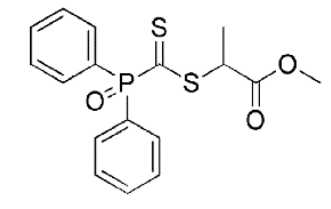

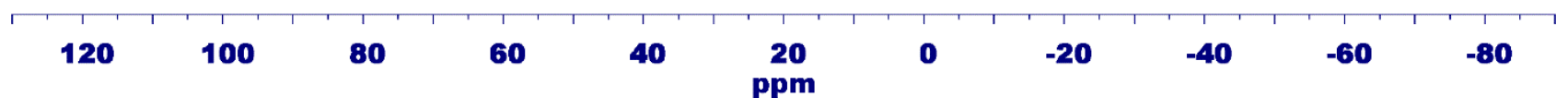

Figure S9. ${ }^{31} \mathrm{P}\left\{{ }^{1} \mathrm{H}\right\}$ NMR spectrum of $S$-((1-methoxycarbonyl)ethyl) (diphenylphosphinoyl)carbodithioate P-RAFT 5.

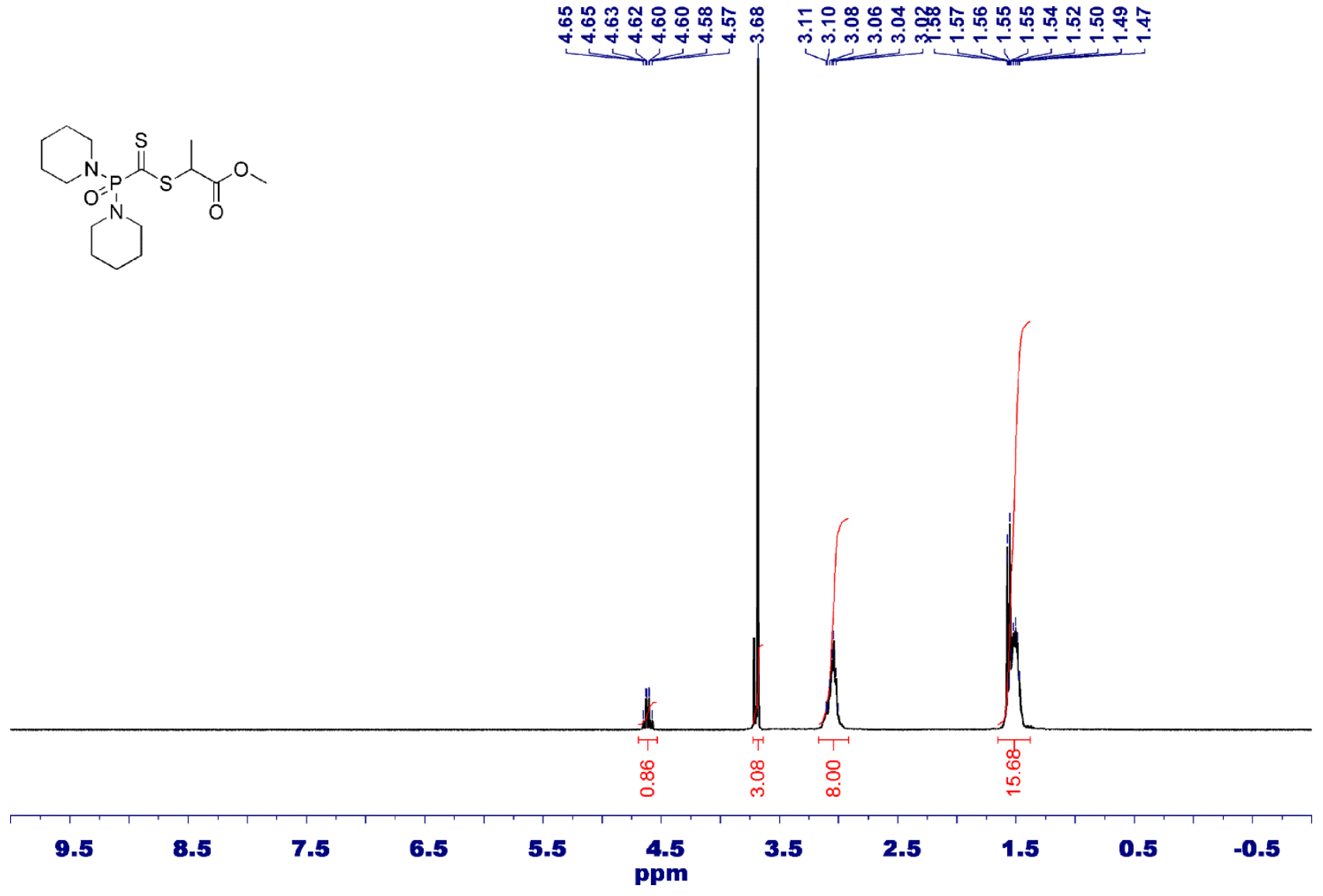


Figure S10. ${ }^{1} \mathrm{H} \quad$ NMR $\quad$ spectrum of methyl $S$-((1-methoxycarbonyl)ethyl) (di(piperidin-1yl)phosphinoyl)carbodithioate P-RAFT 6.

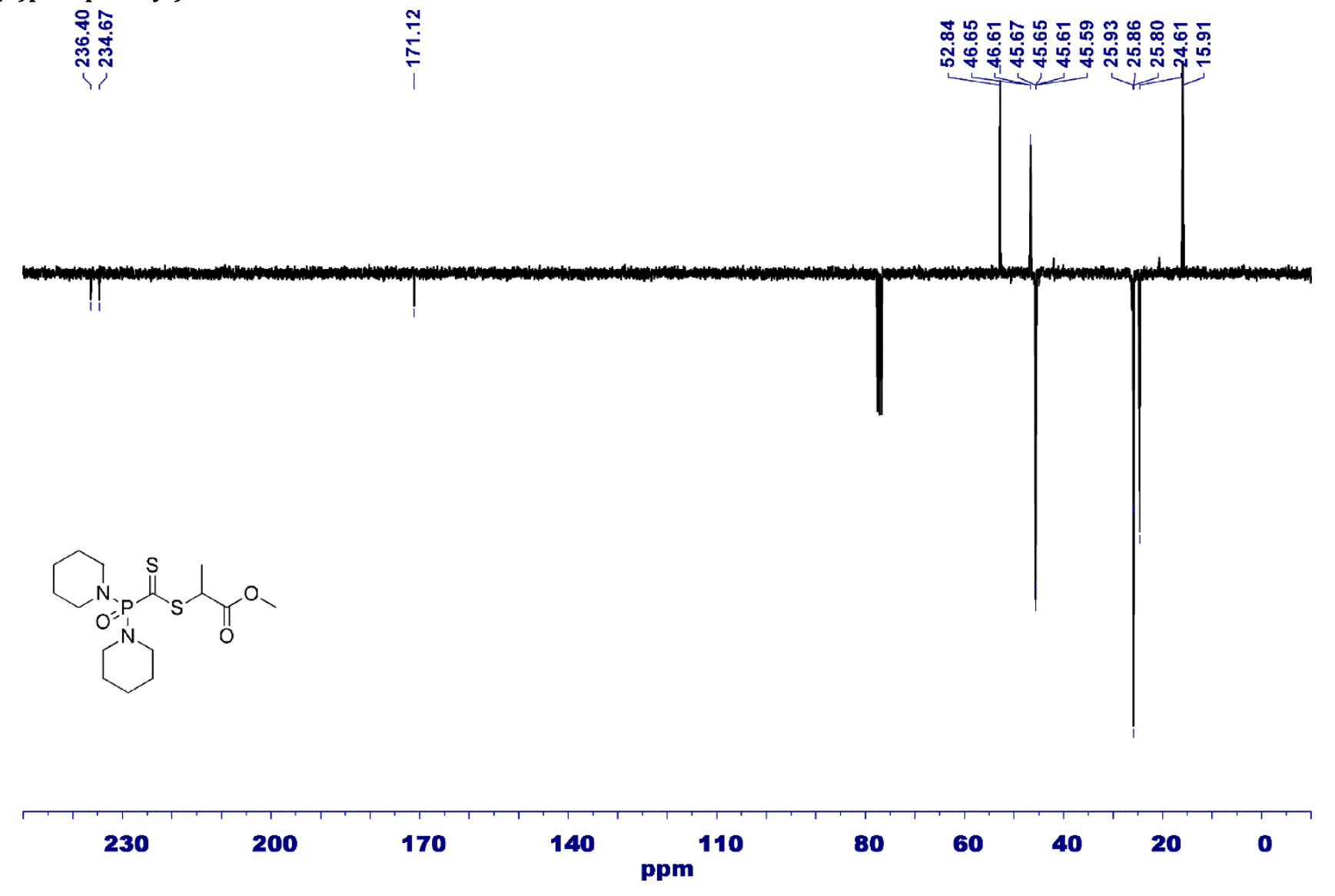

Figure S11. ${ }^{13} \mathrm{C}\left\{{ }^{1} \mathrm{H}\right\} \quad$ NMR spectrum of methyl $S$-((1-methoxycarbonyl)ethyl) (di(piperidin-1yl)phosphinoyl)carbodithioate P-RAFT 6. 


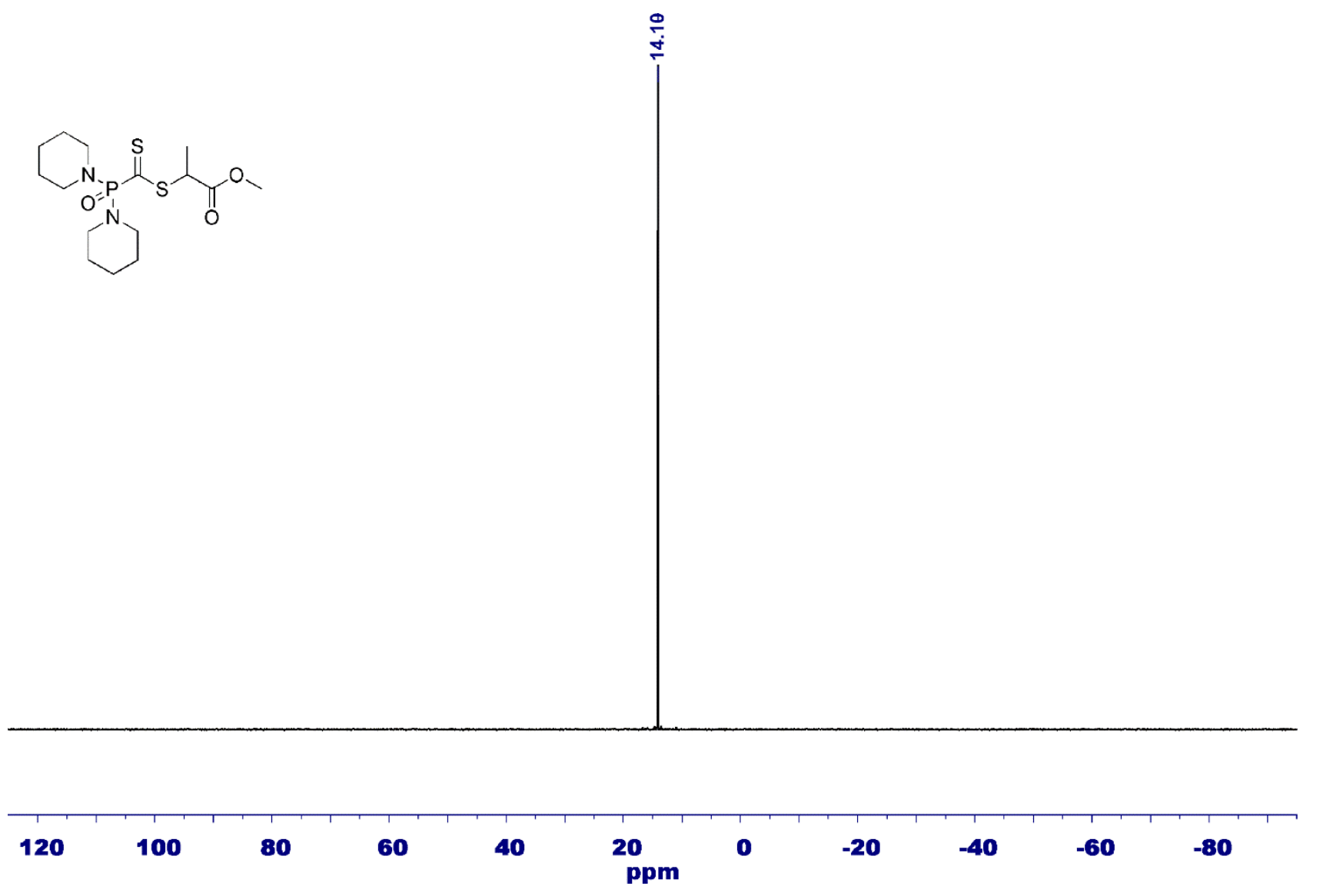

Figure S12. ${ }^{31} \mathrm{P}\left\{{ }^{1} \mathrm{H}\right\} \quad$ NMR spectrum of methyl $S$-((1-methoxycarbonyl)ethyl) (di(piperidin-1yl)phosphinoyl)carbodithioate P-RAFT 6.

Table S2. Macromolecular characteristics of PBA samples obtained during P-RAFT mediated polymerization

\begin{tabular}{|c|c|c|c|c|c|c|c|}
\hline Entry & P-RAFT & $\mathbf{t}, \mathbf{h}$ & $\begin{array}{l}\text { BA } \\
\text { conversion }^{a}\end{array}$ & $\mathrm{CEF}^{b}$ & $M_{\mathrm{nth}}^{c}$ & $M_{\mathrm{n}}{ }^{d}$ & $\boldsymbol{D}^{e}$ \\
\hline 1 & 1 & 4 & $4.2 \%$ & $99 \%$ & 1190 & 1070 & 1.19 \\
\hline 2 & 1 & 9 & $18.5 \%$ & $85 \%$ & 3990 & 5090 & 1.14 \\
\hline 3 & 1 & 13 & $33.3 \%$ & $80 \%$ & 6870 & 8340 & 1.14 \\
\hline 4 & 1 & 24 & $53.5 \%$ & $65 \%$ & 10830 & 14250 & 1.22 \\
\hline 5 & 1 & 36 & $74.3 \%$ & $55 \%$ & 14870 & 21630 & 1.29 \\
\hline 6 & 2 & 2 & $33.7 \%$ & $76 \%$ & 5990 & 6030 & 1.27 \\
\hline 7 & 2 & 3 & $50.9 \%$ & $63 \%$ & 8830 & 9030 & 1.25 \\
\hline 8 & 2 & 6 & $75.4 \%$ & $44 \%$ & 12880 & 14810 & 1.29 \\
\hline 9 & 2 & 9 & $86.2 \%$ & $29 \%$ & 14660 & 17490 & 1.38 \\
\hline 10 & 2 & 13 & $98.5 \%$ & $12 \%$ & 16700 & 18300 & 1.44 \\
\hline 11 & 3 & 2 & $17.4 \%$ & $100 \%$ & 3810 & 4120 & 1.18 \\
\hline 12 & 3 & 4 & $46.9 \%$ & $98 \%$ & 9610 & 11520 & 1.10 \\
\hline 13 & 3 & 9 & $68.0 \%$ & $95 \%$ & 13750 & 15810 & 1.12 \\
\hline 14 & 3 & 13 & $81.2 \%$ & $93 \%$ & 16350 & 18740 & 1.15 \\
\hline 15 & 3 & 24 & $93.9 \%$ & $90 \%$ & 18840 & 22230 & 1.15 \\
\hline 16 & 4 & 3 & $8.5 \%$ & $100 \%$ & 1800 & 2060 & 1.18 \\
\hline 17 & 4 & 5.2 & $20.4 \%$ & $99 \%$ & 3770 & 4760 & 1.16 \\
\hline 18 & 4 & 9 & $37.9 \%$ & $97 \%$ & 6670 & 6930 & 1.13 \\
\hline 19 & 4 & 14 & $62.0 \%$ & $96 \%$ & 10660 & 14340 & 1.14 \\
\hline
\end{tabular}




\begin{tabular}{|c|c|c|c|c|c|c|c|}
\hline 20 & 4 & 36 & $91.1 \%$ & $86 \%$ & 15470 & 21120 & 1.18 \\
\hline 21 & 5 & 6 & $16.7 \%$ & $100 \%$ & 3650 & 4700 & 1.12 \\
\hline 22 & 5 & 9 & $24.4 \%$ & $94 \%$ & 5160 & 7180 & 1.11 \\
\hline 23 & 5 & 13 & $36.7 \%$ & $85 \%$ & 7580 & 10600 & 1.16 \\
\hline 24 & 5 & 24 & $54.7 \%$ & $67 \%$ & 11110 & 16160 & 1.29 \\
\hline 25 & 5 & 36 & $73.5 \%$ & $46 \%$ & 14810 & 22370 & 1.45 \\
\hline 26 & 6 & 3 & $20.2 \%$ & $91 \%$ & 4330 & 4700 & 1.17 \\
\hline 27 & 6 & 6 & $38.9 \%$ & $91 \%$ & 7980 & 9950 & 1.10 \\
\hline 28 & 6 & 9 & $51.5 \%$ & $90 \%$ & 10190 & 13310 & 1.12 \\
\hline 29 & 6 & 13 & $64.8 \%$ & $88 \%$ & 13050 & 16760 & 1.15 \\
\hline 30 & 6 & 24 & $80.3 \%$ & $82 \%$ & 16080 & 21700 & 1.19 \\
\hline
\end{tabular}

${ }^{a}$ Determined by ${ }^{1} \mathrm{H}$ NMR.

${ }^{b}$ Chain end fidelity determined by ${ }^{31} \mathrm{P}\left\{{ }^{1} \mathrm{H}\right\} \mathrm{NMR}$.

${ }^{c} M_{\mathrm{n} \text { th }}=M_{\mathrm{w}}(\mathrm{P}-\mathrm{RAFT})+\left([\mathrm{BA}]_{0} /[\mathrm{P}-\mathrm{RAFT}]_{0}\right) \times$ conversion $\times M_{\mathrm{w}}(\mathrm{BA})$.

${ }^{d}$ Determined by SEC.

${ }^{e} \bigoplus=M_{\mathrm{w}} / M_{\mathrm{n}}$.

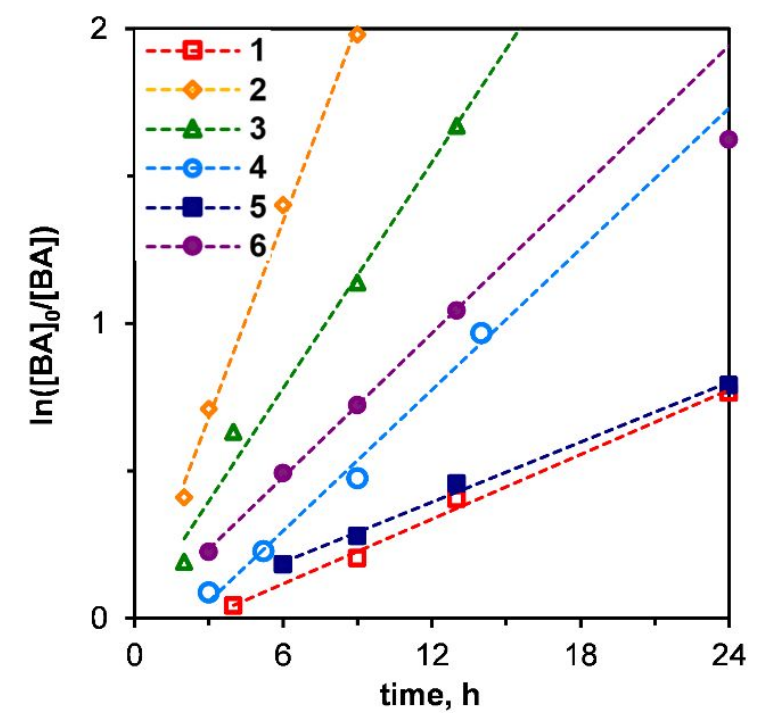

Figure S13. Semi-logarithmic kinetic plot for the initial period

P-RAFT 1-6 mediated BA polymerization.

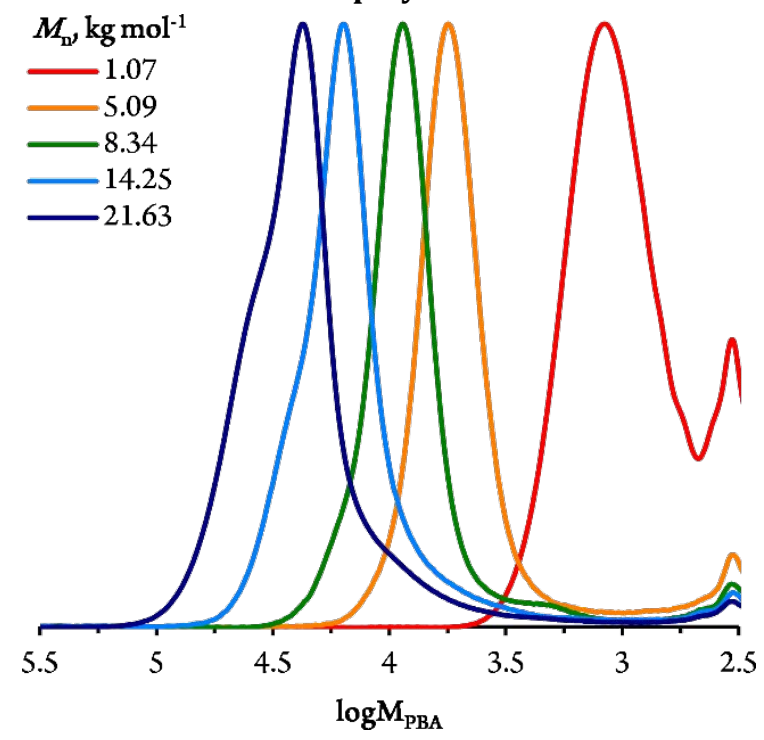

Figure S14. Overlay of the SEC chromatograms of PBA samples obtained in P-RAFT 1 mediated polymerization.

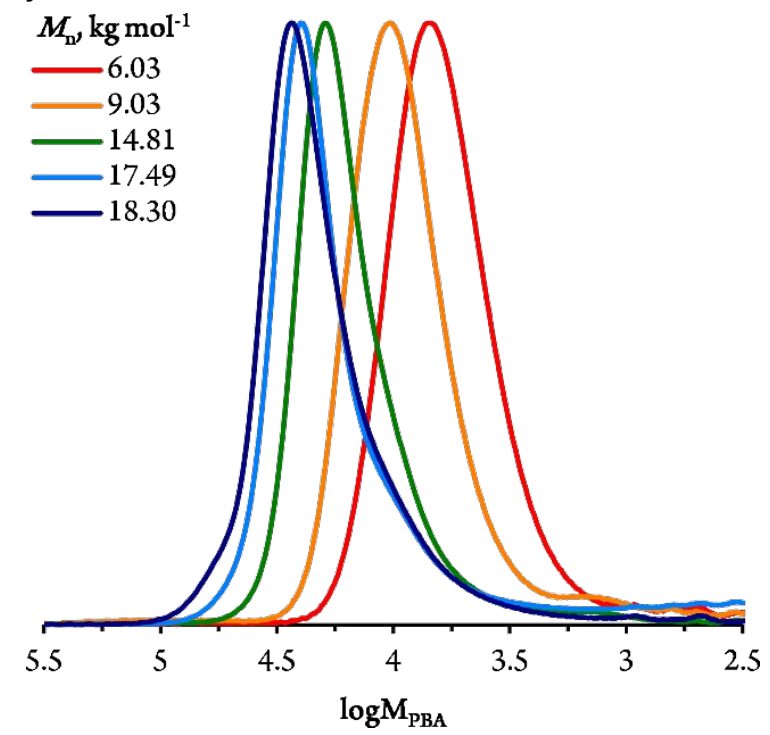

Figure S15. Overlay of the SEC chromatograms of PBA samples obtained in P-RAFT 2 mediated polymerization. 


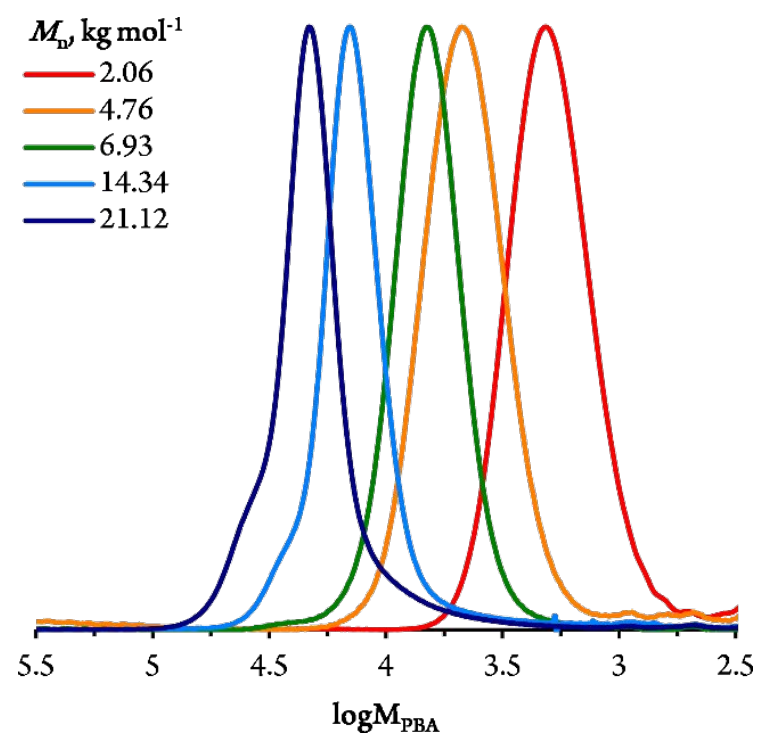

Figure S16. Overlay of the SEC chromatograms of PBA samples obtained in P-RAFT 4 mediated polymerization.

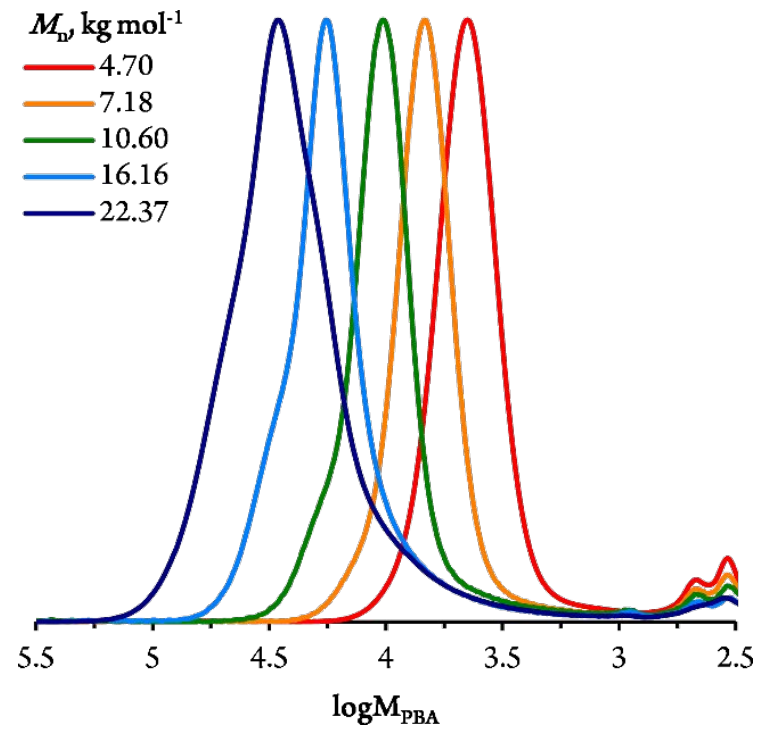

Figure S17. Overlay of the SEC chromatograms of PBA samples obtained in P-RAFT 5 mediated polymerization.

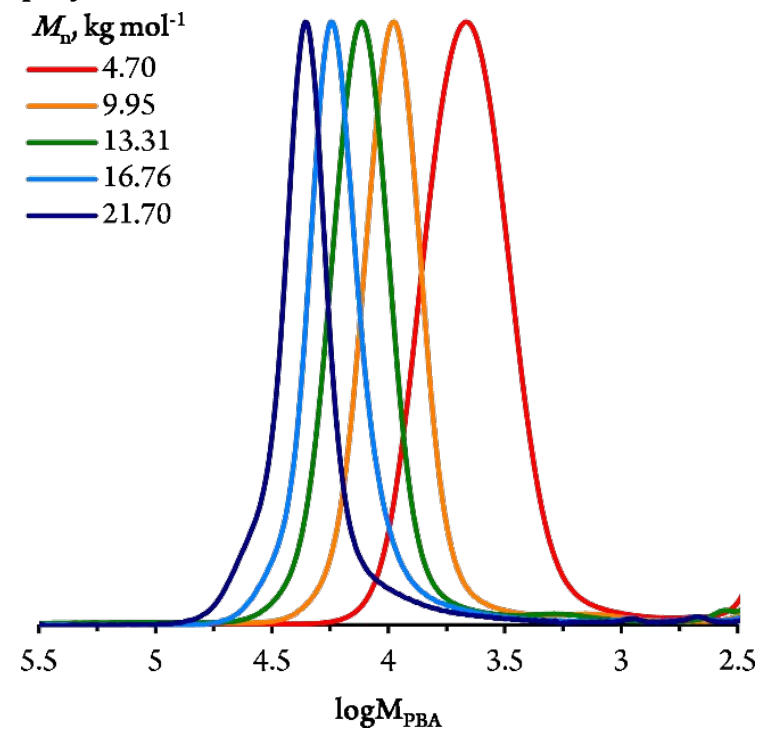

Figure S18. Overlay of the SEC chromatograms of PBA samples obtained in P-RAFT 6 mediated polymerization. 


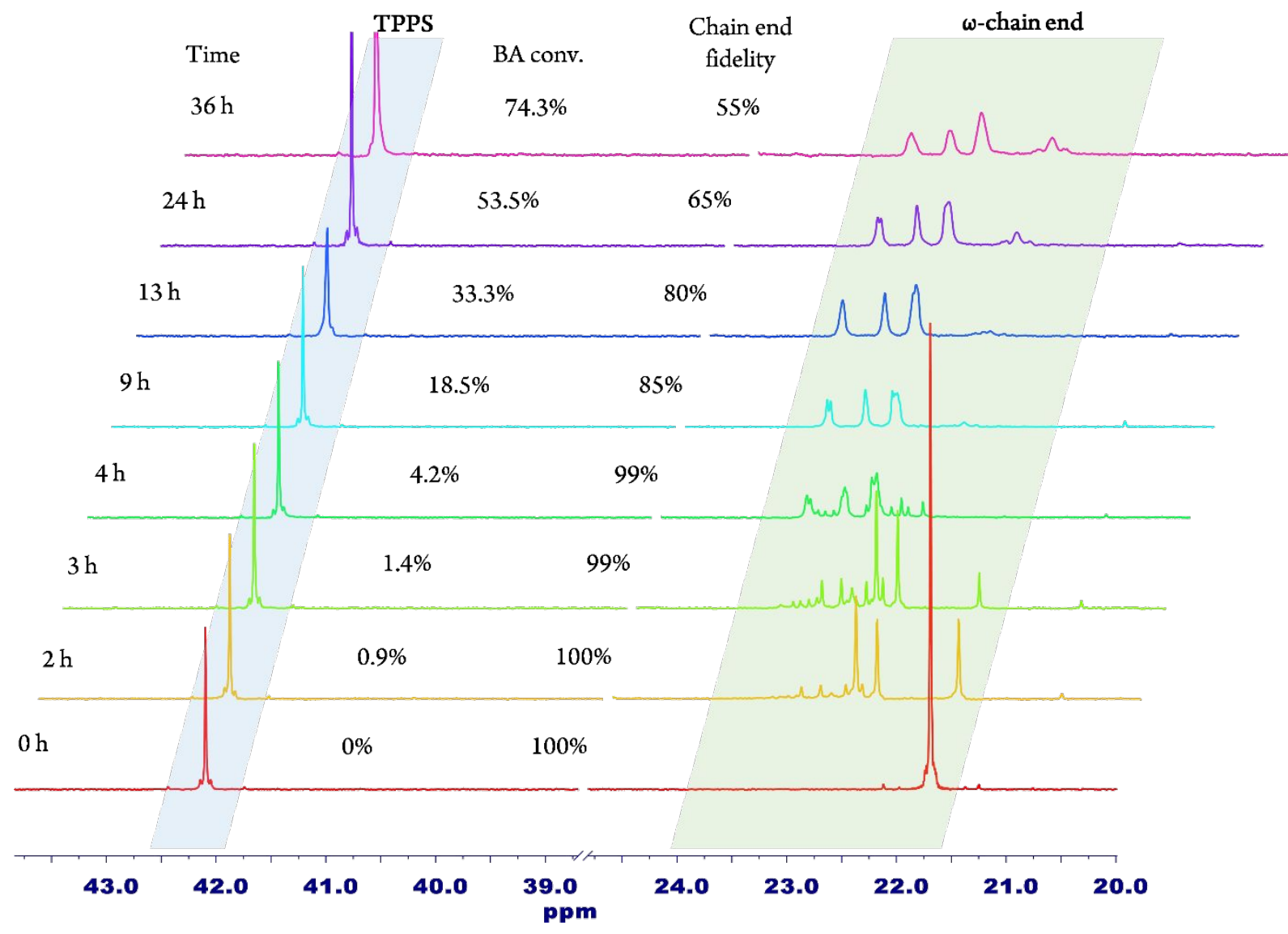

Figure S19. ${ }^{31} \mathrm{P}\left\{{ }^{1} \mathrm{H}\right\}$ NMR spectra of reaction mixtures obtained in P-RAFT 1 mediated polymerization of BA.

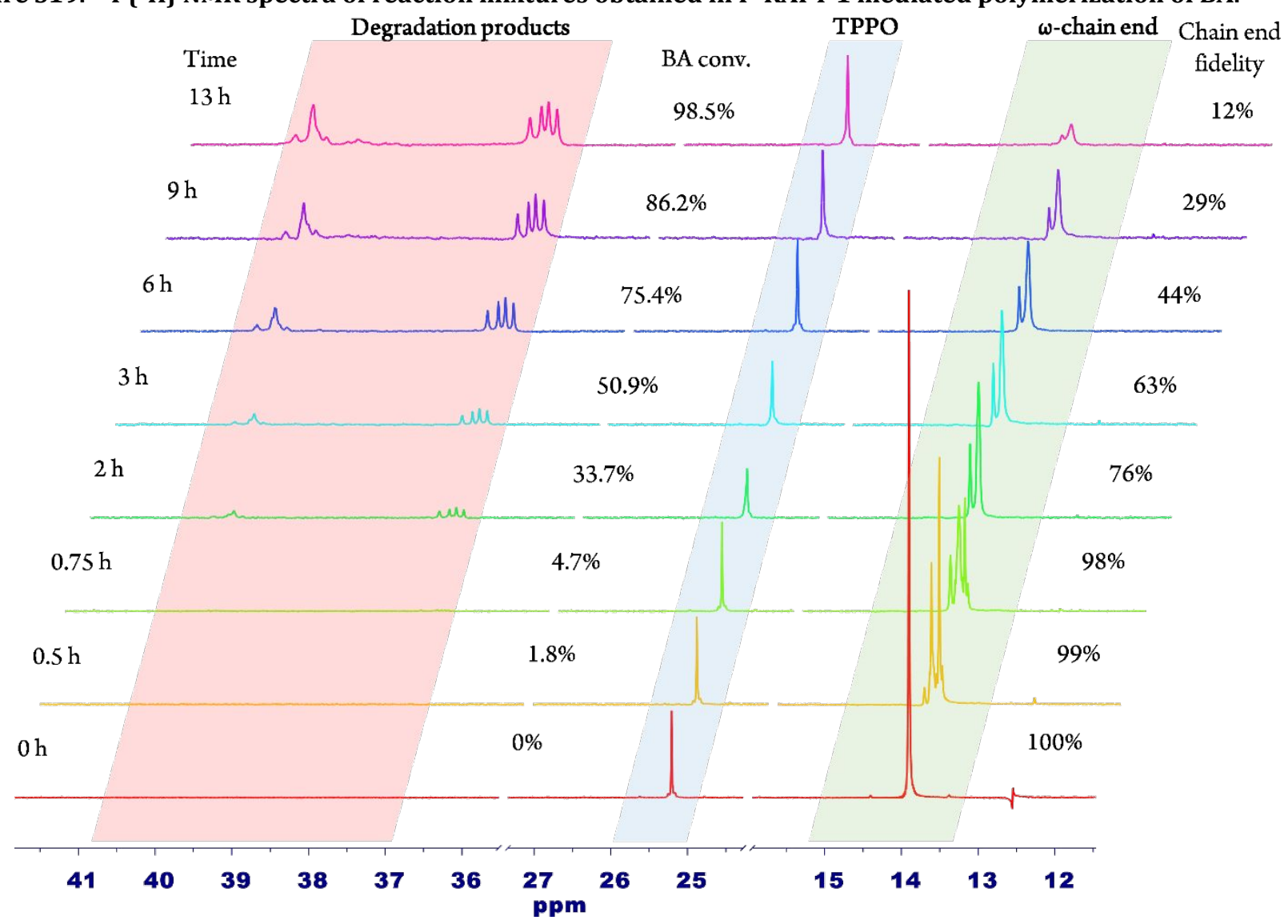

Figure S20. ${ }^{31} \mathrm{P}\left\{{ }^{1} \mathrm{H}\right\}$ NMR spectra of reaction mixtures obtained in P-RAFT 2 mediated polymerization of BA. 


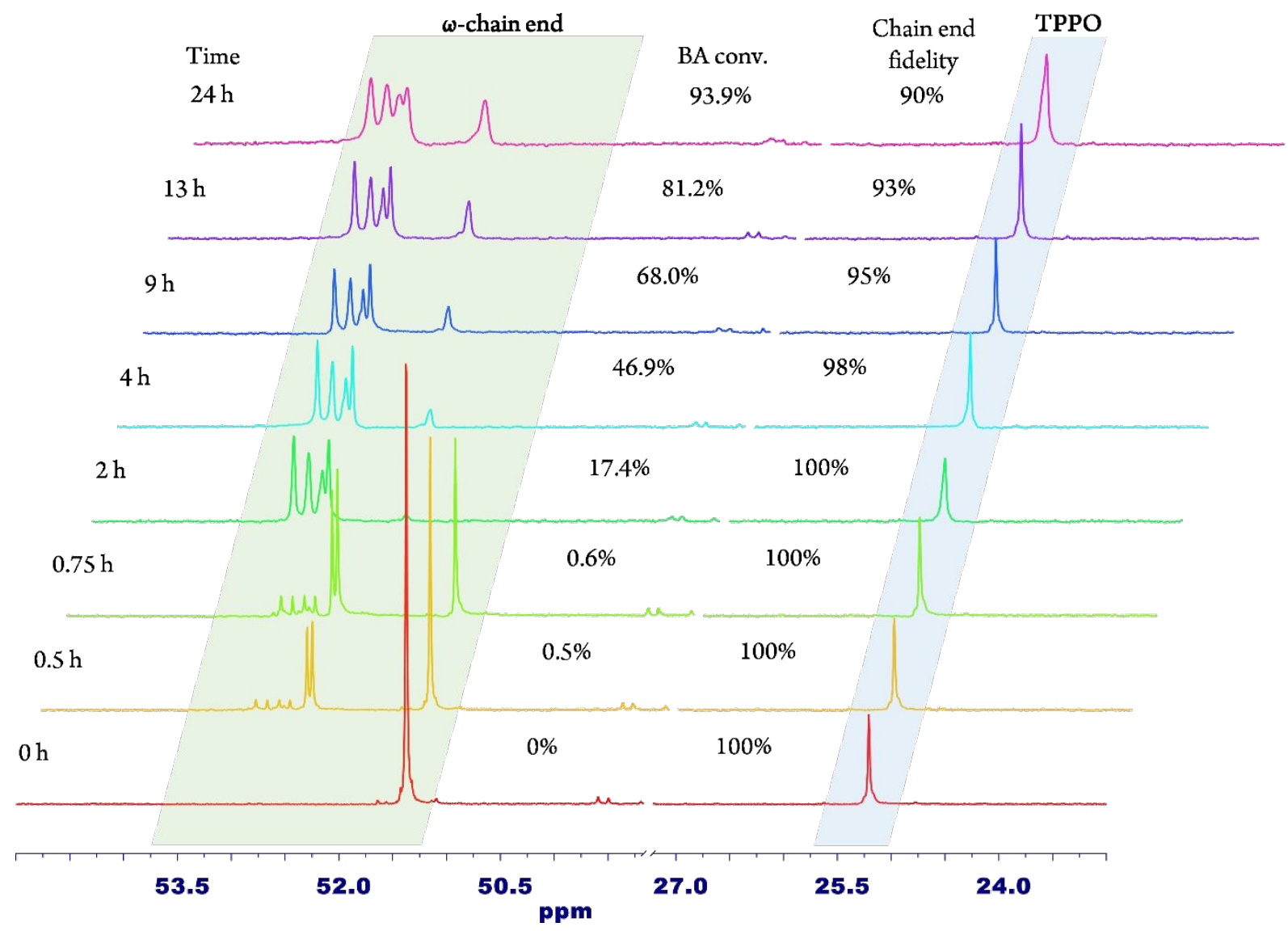

Figure S21. ${ }^{31} \mathrm{P}\left\{{ }^{1} \mathrm{H}\right\}$ NMR spectra of reaction mixtures obtained in P-RAFT 3 mediated polymerization of BA.

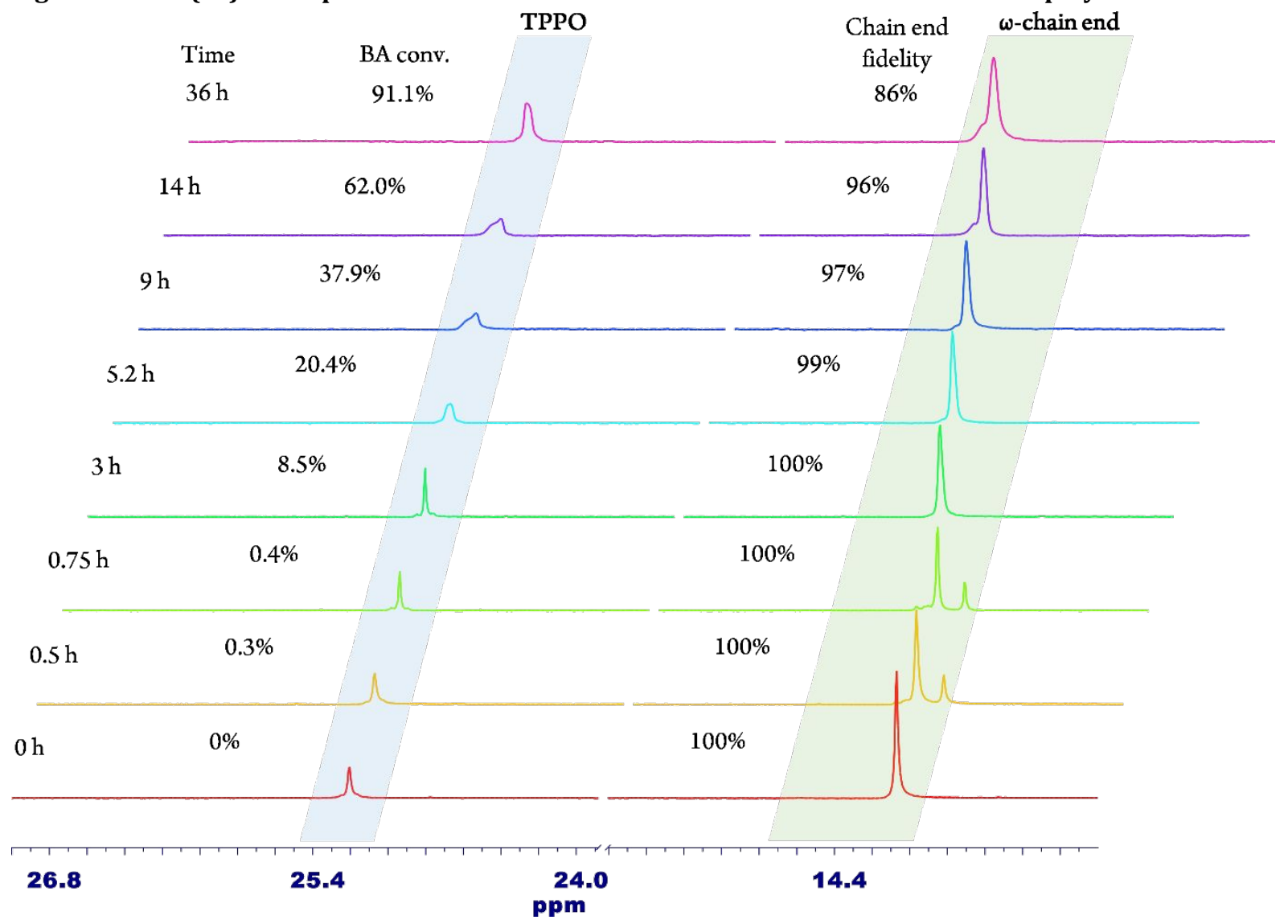

Figure S22. ${ }^{31} \mathrm{P}\left\{{ }^{1} \mathrm{H}\right\}$ NMR spectra of reaction mixtures obtained in P-RAFT 4 mediated polymerization of BA. 


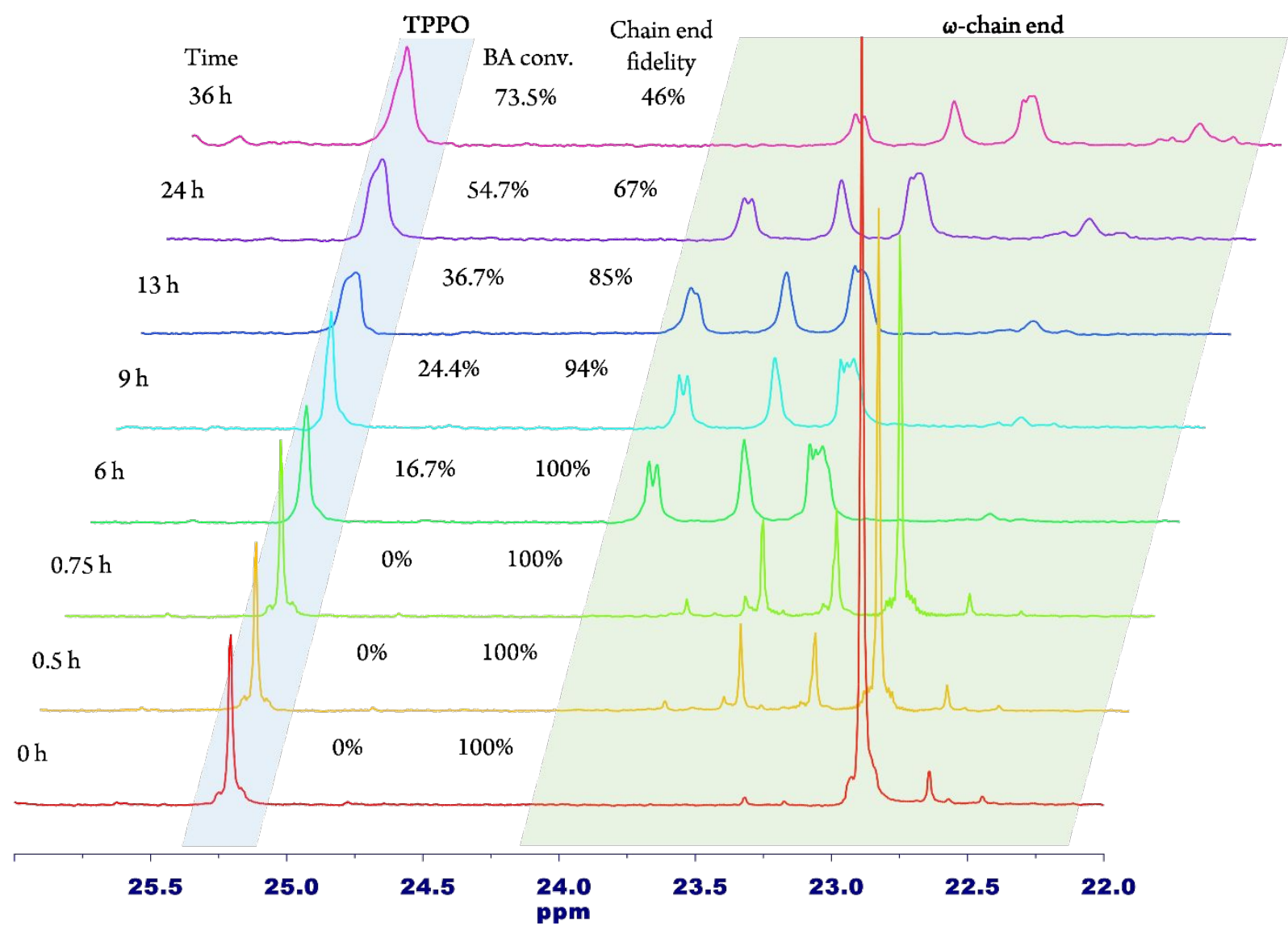

Figure S23. ${ }^{31} \mathrm{P}\left\{{ }^{1} \mathrm{H}\right\}$ NMR spectra of reaction mixtures obtained in P-RAFT 5 mediated polymerization of BA.

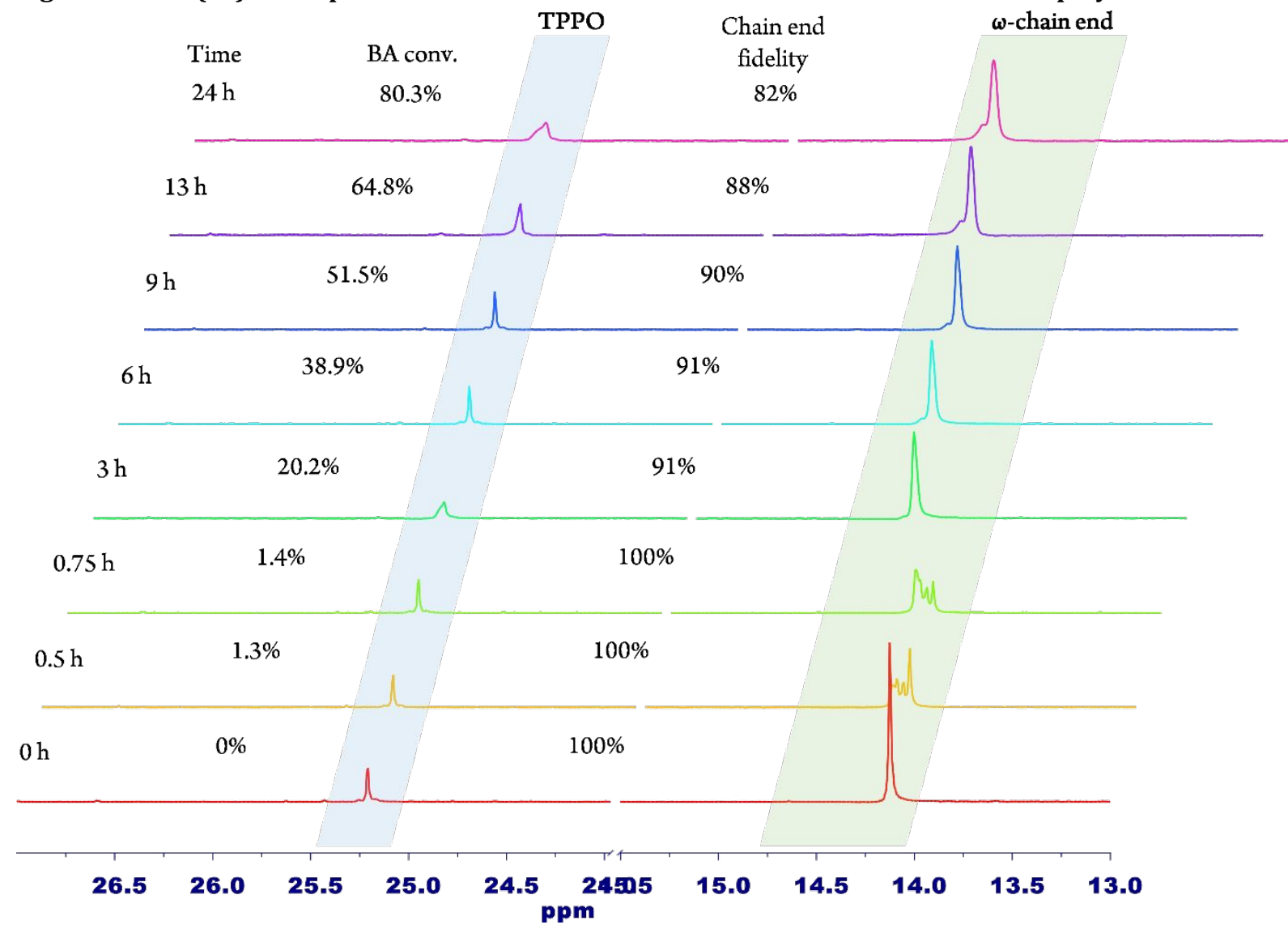

Figure S24. ${ }^{31} \mathrm{P}\left\{{ }^{1} \mathrm{H}\right\}$ NMR spectra of reaction mixtures obtained in P-RAFT 6 mediated polymerization of BA. 

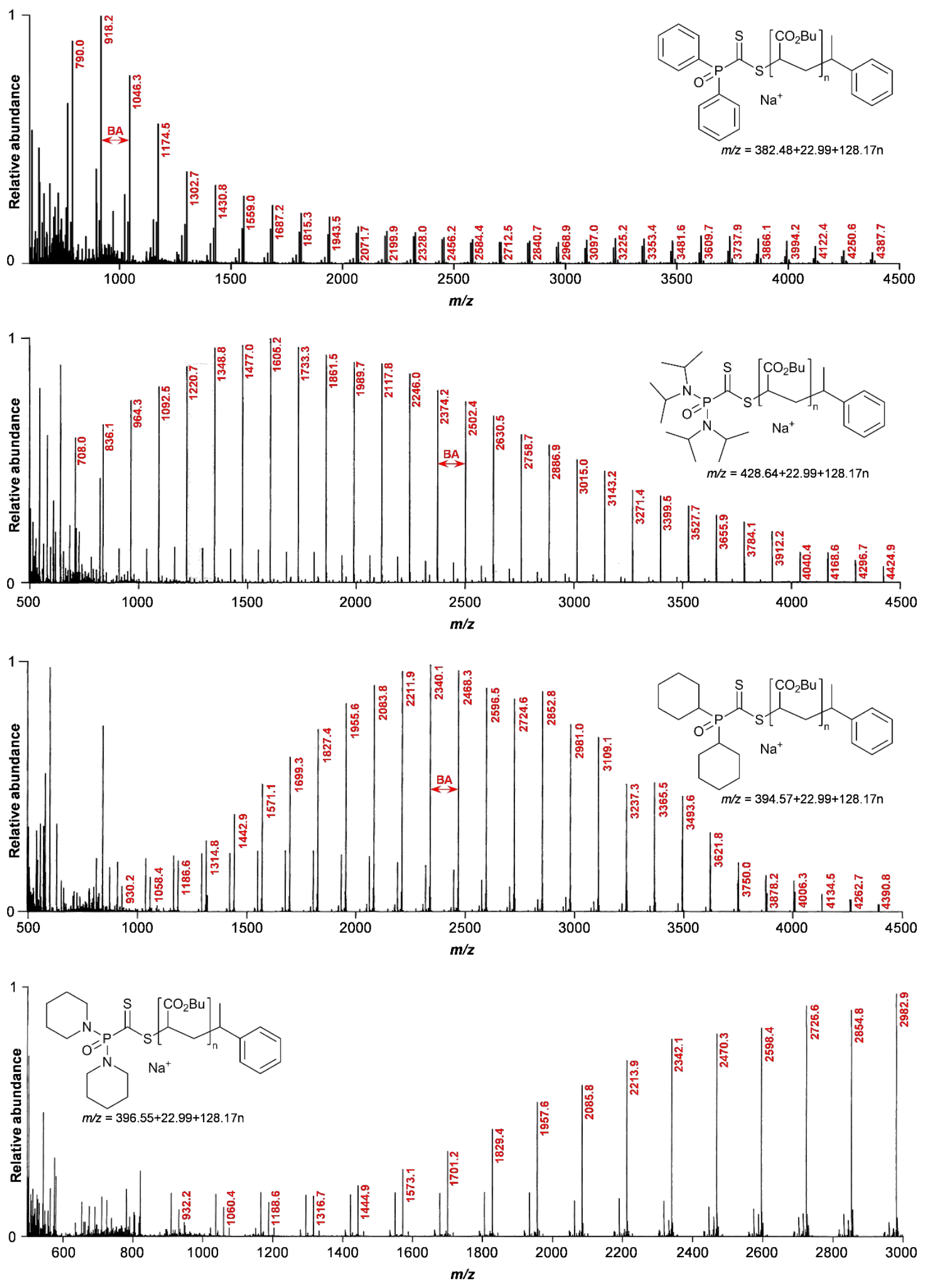

Figure S25. MALDI-TOF MS spectra of PBA samples prepared starting from P-RAFT 1-4. 
Table S3. Macromolecular characteristics of PSt samples obtained in P-RAFT mediated polymerization

\begin{tabular}{|c|c|c|c|c|c|c|c|}
\hline Entry & P-RAFT & $\mathbf{t}, \mathbf{h}$ & $\begin{array}{l}\text { St } \\
\text { conversion }^{a}\end{array}$ & $\mathrm{CEF}^{b}$ & $M_{\mathrm{n} \mathrm{th}^{c}}$ & $M_{\mathrm{n}}{ }^{d}$ & $\boldsymbol{\varpi}^{e}$ \\
\hline 31 & 1 & 5.5 & $5.2 \%$ & $97 \%$ & 1140 & 1030 & 1.12 \\
\hline 32 & 1 & 15 & $18.9 \%$ & $92 \%$ & 3140 & 3050 & 1.11 \\
\hline 33 & 1 & 23 & $26.8 \%$ & $85 \%$ & 4300 & 4360 & 1.14 \\
\hline 34 & 1 & 48 & $44.4 \%$ & $77 \%$ & 6860 & 7490 & 1.16 \\
\hline 35 & 1 & 110 & $55.0 \%$ & $55 \%$ & 8410 & 8840 & 1.22 \\
\hline 36 & 2 & 5.5 & $11.7 \%$ & $100 \%$ & 2090 & 1340 & 1.12 \\
\hline 37 & 2 & 17 & $25.9 \%$ & $99 \%$ & 4110 & 4100 & 1.07 \\
\hline 38 & 2 & 27 & $38.2 \%$ & $99 \%$ & 5860 & 5900 & 1.07 \\
\hline 39 & 2 & 50 & $57.2 \%$ & $99 \%$ & 8560 & 8410 & 1.06 \\
\hline 40 & 2 & 100 & $78.6 \%$ & $99 \%$ & 11610 & 12590 & 1.08 \\
\hline 41 & 3 & 5.5 & $9.2 \%$ & $98 \%$ & 1920 & 1300 & 1.08 \\
\hline 42 & 3 & 15 & $23.5 \%$ & $98 \%$ & 4300 & 4180 & 1.09 \\
\hline 43 & 3 & 23 & $34.3 \%$ & $98 \%$ & 6100 & 6420 & 1.10 \\
\hline 44 & 3 & 48 & $52.2 \%$ & $98 \%$ & 9560 & 9460 & 1.14 \\
\hline 45 & 3 & 110 & $76.2 \%$ & $98 \%$ & 12600 & 13220 & 1.14 \\
\hline 46 & 4 & 5.5 & $9.6 \%$ & $98 \%$ & 1990 & 1380 & 1.13 \\
\hline 47 & 4 & 15 & $23.8 \%$ & $98 \%$ & 4340 & 4390 & 1.08 \\
\hline 48 & 4 & 23 & $34.4 \%$ & $97 \%$ & 6090 & 6290 & 1.09 \\
\hline 49 & 4 & 48 & $55.1 \%$ & $96 \%$ & 9520 & 10470 & 1.11 \\
\hline 50 & 4 & 110 & $73.4 \%$ & $96 \%$ & 12550 & 13850 & 1.13 \\
\hline 51 & PBA-3 & 0 & $0.0 \%$ & $100 \%$ & 2270 & 2270 & 1.25 \\
\hline 52 & PBA-3 & 5.5 & $9.4 \%$ & $97 \%$ & 4600 & 4440 & 1.16 \\
\hline 53 & PBA-3 & 15 & $27.5 \%$ & $94 \%$ & 9090 & 8690 & 1.13 \\
\hline 54 & PBA-3 & 23 & $36.8 \%$ & $93 \%$ & 11380 & 10700 & 1.15 \\
\hline 55 & PBA-3 & 48 & $54.9 \%$ & $93 \%$ & 15880 & 15750 & 1.16 \\
\hline 56 & PBA-3 & 110 & $74.3 \%$ & $93 \%$ & 20690 & 21040 & 1.13 \\
\hline
\end{tabular}




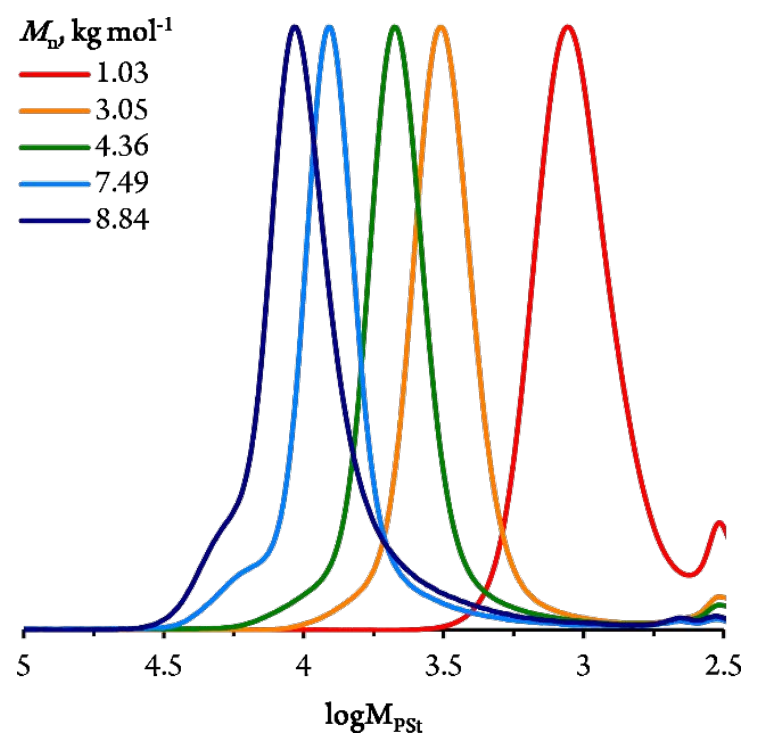

Figure S26. Overlay of the SEC chromatograms of PSt samples obtained in P-RAFT 1 mediated polymerization.

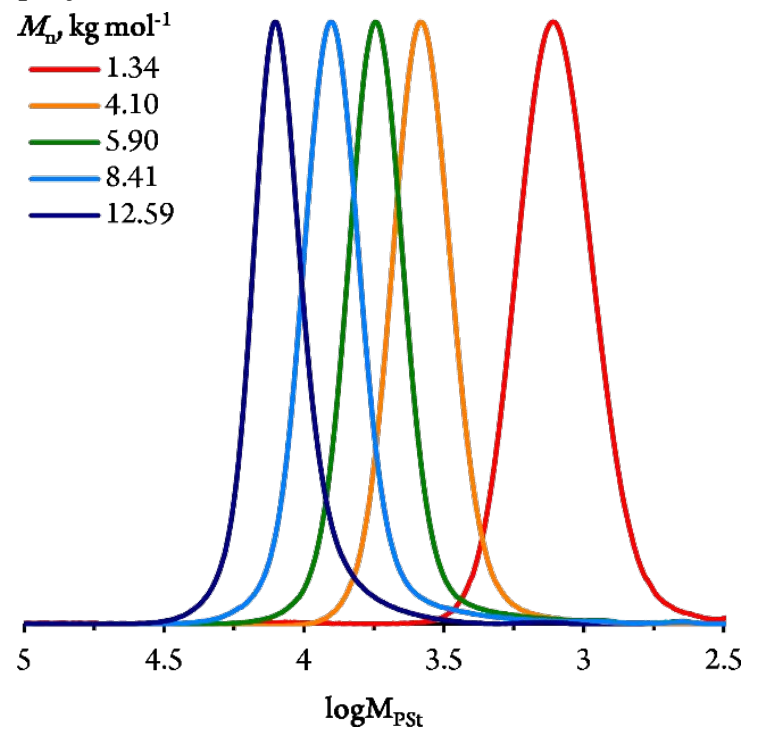

Figure S27. Overlay of the SEC chromatograms of PSt samples obtained in P-RAFT 2 mediated polymerization.

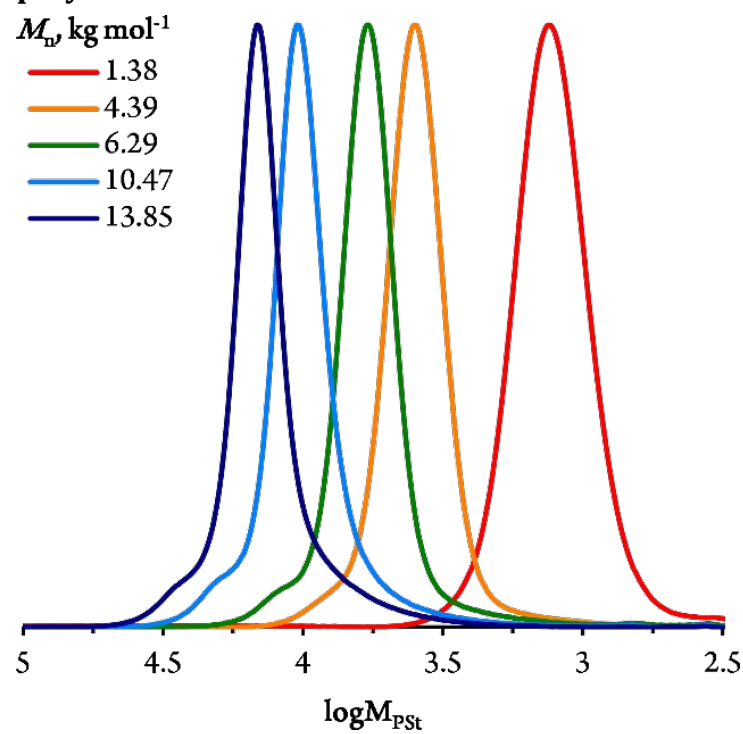

Figure S28. Overlay of the SEC chromatograms of PSt samples obtained in P-RAFT 4 mediated polymerization. 


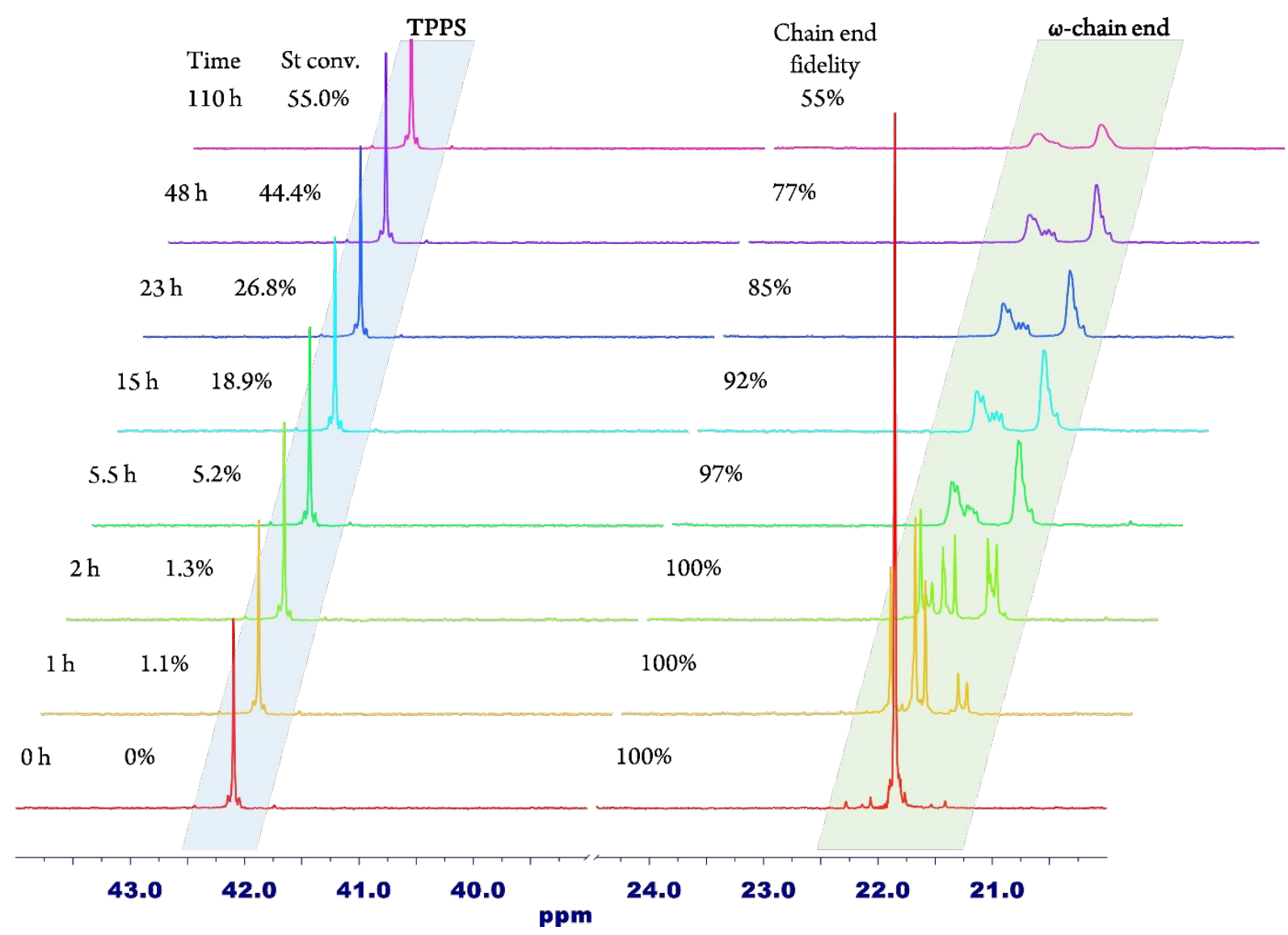

Figure S29. ${ }^{31} \mathrm{P}\left\{{ }^{1} \mathrm{H}\right\}$ NMR spectra of reaction mixtures obtained in P-RAFT 1 mediated polymerization of St.

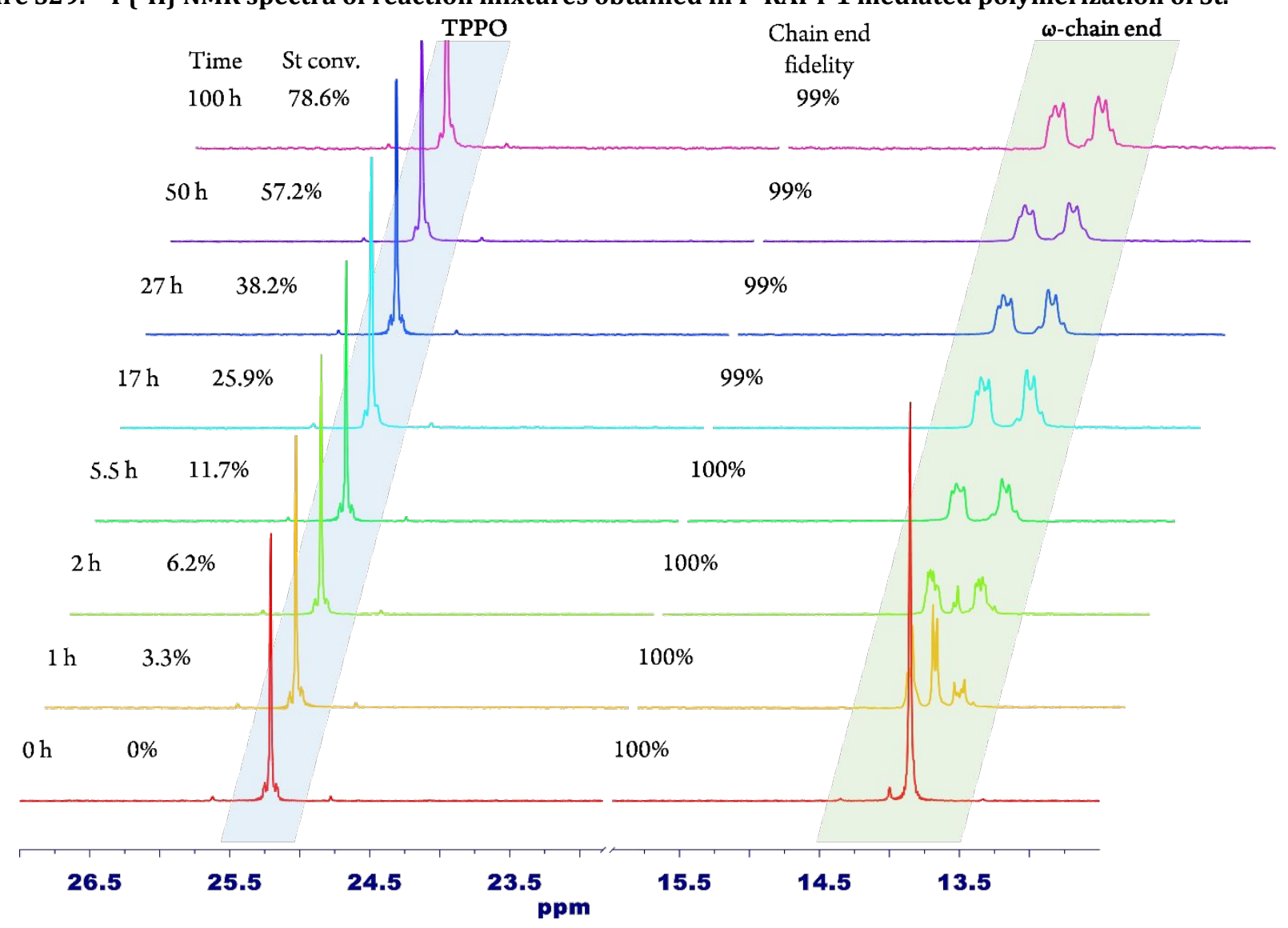

Figure S30. ${ }^{31} \mathrm{P}\left\{{ }^{1} \mathrm{H}\right\}$ NMR spectra of reaction mixtures obtained in P-RAFT 2 mediated polymerization of St. 


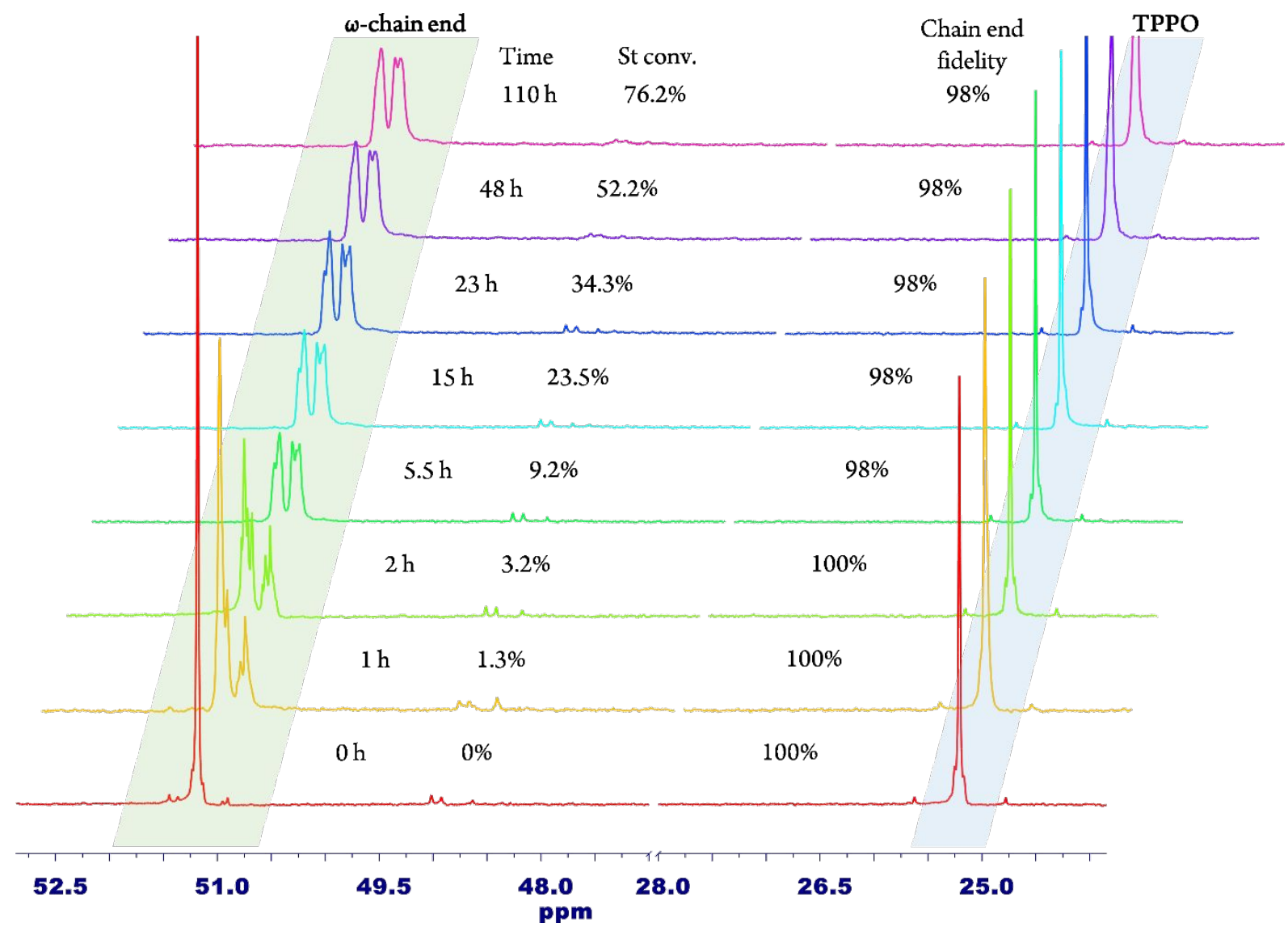

Figure S31. ${ }^{31} \mathrm{P}\left\{{ }^{1} \mathrm{H}\right\}$ NMR spectra of reaction mixtures obtained in P-RAFT 3 mediated polymerization of St.

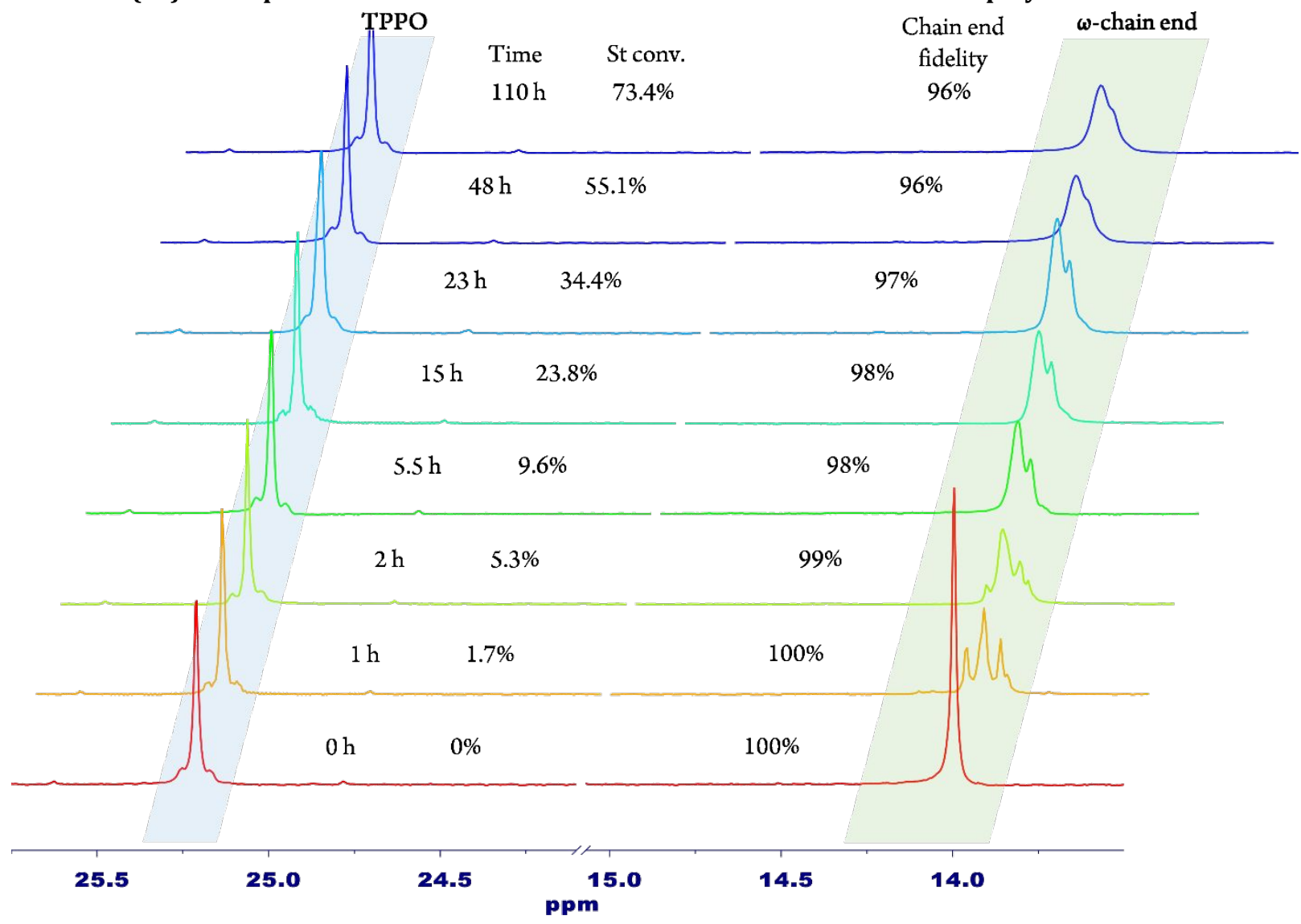

Figure S32. ${ }^{31} \mathrm{P}\left\{{ }^{1} \mathrm{H}\right\}$ NMR spectra of reaction mixtures obtained in P-RAFT 4 mediated polymerization of St. 


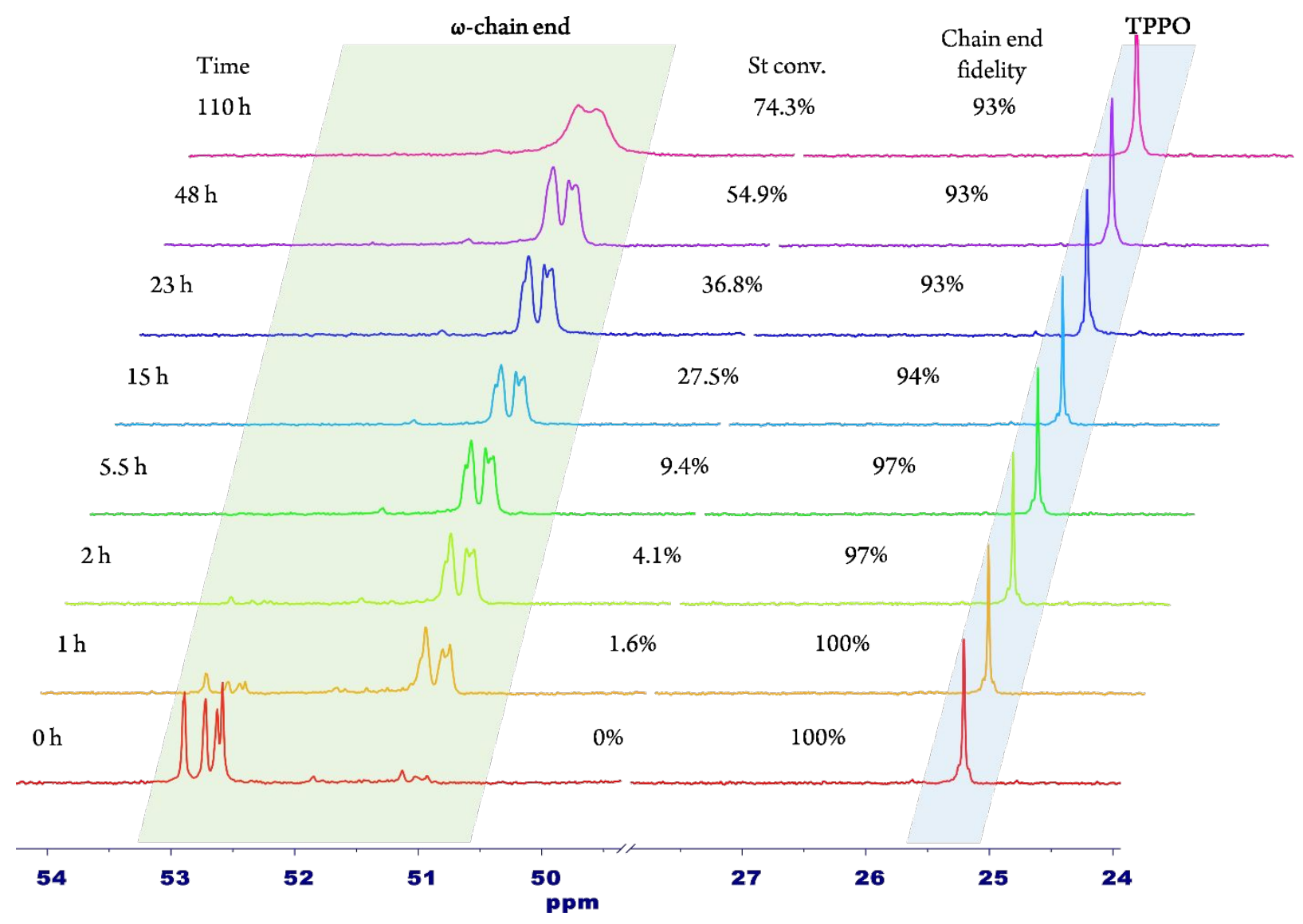

Figure S33. ${ }^{31} \mathrm{P}\left\{{ }^{1} \mathrm{H}\right\}$ NMR spectra of reaction mixtures obtained in PBA-3 mediated polymerization of St.

Table S4. ${ }^{31} \mathrm{P}\left\{{ }^{1} \mathrm{H}\right\}$ NMR chemical shifts used to determine the RAFT $\omega$ chain-end fidelity ${ }^{\mathrm{a}}$

\begin{tabular}{llllll}
\hline $\begin{array}{l}\text { Entries of } \\
\text { Tables } \mathbf{S 2} \\
\text { and S3 }\end{array}$ & P-RAFT & Internal standard & Polymer & \multicolumn{2}{c}{ Chemical shift (ppm) } \\
\hline $1-5$ & $\mathbf{1}$ & TPPS & & w-chain end & Internal standard \\
$6-10$ & $\mathbf{2}$ & TPPO & PBA & $24.0-21.5$ & 42.1 \\
$11-15$ & $\mathbf{3}$ & TPPO & PBA & $14.5-13.5$ & 25.5 \\
$16-20$ & $\mathbf{4}$ & TPPO & PBA & $53.8-51.2$ & 25.5 \\
$21-25$ & $\mathbf{5}$ & TPPO & PBA & $14.8-14.0$ & 25.5 \\
$26-30$ & $\mathbf{6}$ & TPPO & PBA & $24.2-22.2$ & 25.5 \\
$31-35$ & $\mathbf{1}$ & TPPS & PSt & $14.8-14.0$ & 25.5 \\
$36-40$ & $\mathbf{2}$ & TPPO & PSt & $14.2-13.5$ & 42.1 \\
$41-45$ & $\mathbf{3}$ & TPPO & PSt & $51.8-50.8$ & 25.5 \\
$46-50$ & $\mathbf{4}$ & TPPO & PSt & $14.2-13.9$ & 25.5 \\
$51-56$ & PBA-3 & TPPO & PBA/PSt & $53.5-50.8$ & 25.5 \\
\hline
\end{tabular}

${ }^{a}$ Chain-end fidelity can be calculated from ${ }^{31} \mathrm{P}\left\{{ }^{1} \mathrm{H}\right\}$ NMR spectra according to the following equation:

$$
C E F=\frac{I_{\omega t} \times I_{I S t_{0}}}{I_{\omega} t_{0} \times I_{I S t}}
$$

$C E F$ - chain-end fidelity;

$I_{\omega t}$ - integral intensity of the signals of the organophosphorus $\omega$-chain end at time $t$;

$I_{\omega t_{0}}$ - integral intensity of the signals of the organophosphorus $\omega$-chain end at time zero;

$I_{I S t}$ - integral intensity of the signals of the internal standard at time $\mathrm{t}$;

$I_{I S} t_{0}$ - integral intensity of the signals of the internal standard at time $t$; 